\title{
बrecreative
}

ISSN 1855-3966 (printed edn.), ISSN 1855-3974 (electronic edn.)

ARS MATHEMATICA CONTEMPORANEA 18 (2020) 241-271

https://doi.org/10.26493/1855-3974.2110.6f2

(Also available at http://amc-journal.eu)

\section{Relative Heffter arrays and biembeddings}

\author{
Simone Costa $(\mathbb{D}$, Anita Pasotti * \\ DICATAM - Sez. Matematica, Università degli Studi di Brescia, \\ Via Branze 43, I-25123 Brescia, Italy \\ Marco Antonio Pellegrini \\ Dipartimento di Matematica e Fisica, Università Cattolica del Sacro Cuore, \\ Via Musei 41, I-25121 Brescia, Italy
}

Received 6 September 2019, accepted 2 March 2020, published online 20 October 2020

\begin{abstract}
Relative Heffter arrays, denoted by $\mathrm{H}_{t}(m, n ; s, k)$, have been introduced as a generalization of the classical concept of Heffter array. $\mathrm{A}_{t}(m, n ; s, k)$ is an $m \times n$ partially filled array with elements in $\mathbb{Z}_{v}$, where $v=2 n k+t$, whose rows contain $s$ filled cells and whose columns contain $k$ filled cells, such that the elements in every row and column sum to zero and, for every $x \in \mathbb{Z}_{v}$ not belonging to the subgroup of order $t$, either $x$ or $-x$ appears in the array. In this paper we show how relative Heffter arrays can be used to construct biembeddings of cyclic cycle decompositions of the complete multipartite graph $K_{\frac{2 n k+t}{t} \times t}$ into an orientable surface. In particular, we construct such biembeddings providing integer globally simple square relative Heffter arrays for $t=k=3,5,7,9$ and $n \equiv 3(\bmod 4)$ and for $k=3$ with $t=n, 2 n$, any odd $n$.
\end{abstract}

Keywords: Heffter array, biembedding, complete multipartite graph.

Math. Subj. Class. (2020): 05B20, 05B30, 05C10

\section{Introduction}

An $m \times n$ partially filled (p.f., for short) array on a set $\Omega$ is an $m \times n$ matrix whose elements belong to $\Omega$ and where we also allow some cells to be empty. The following class of p.f. arrays was introduced in [15], generalizing the ideas of [2]:

\footnotetext{
* Corresponding author.

E-mail addresses: simone.costa@unibs.it (Simone Costa), anita.pasotti@unibs.it (Anita Pasotti), marcoantonio.pellegrini@unicatt.it (Marco Antonio Pellegrini)
} 
Definition 1.1. Let $v=2 n k+t$ be a positive integer and let $J$ be the subgroup of $\mathbb{Z}_{v}$ of order $t . \mathrm{A}_{t}(m, n ; s, k)$ Heffter array over $\mathbb{Z}_{v}$ relative to $J$ is an $m \times n$ p.f. array with elements in $\mathbb{Z}_{v}$ such that:

(a) each row contains $s$ filled cells and each column contains $k$ filled cells;

(b) for every $x \in \mathbb{Z}_{2 n k+t} \backslash J$, either $x$ or $-x$ appears in the array;

(c) the elements in every row and column sum to zero.

Trivial necessary conditions for the existence of a $\mathrm{H}_{t}(m, n ; s, k)$ are that $t$ divides $2 n k$, $n k=m s, 3 \leq s \leq n$ and $3 \leq k \leq m$. If $\mathrm{H}_{t}(m, n ; s, k)$ is a square array, it will be denoted by $\mathrm{H}_{t}(n ; k)$. A relative Heffter array is called integer if Condition (c) in Definition 1.1 is strengthened so that the elements in every row and in every column, viewed as integers in $\pm\left\{1, \ldots,\left\lfloor\frac{2 n k+t}{2}\right\rfloor\right\}$, sum to zero in $\mathbb{Z}$. We remark that, if $t=1$, namely if $J$ is the trivial subgroup of $\mathbb{Z}_{2 n k+1}$, we find again the classical concept of a (integer) Heffter array, see $[2,3,4,9,10,13,16,17]$. In particular, in [10] it was proved that Heffter arrays $\mathrm{H}_{1}(n ; k)$ exist for all $n \geq k \geq 3$, while by $[4,17]$ integer Heffter arrays $\mathrm{H}_{1}(n ; k)$ exist if and only if the additional condition $n k \equiv 0,3(\bmod 4)$ holds. At the moment, the only known results concerning relative Heffter arrays are described in [15, 22]. Some necessary conditions for the existence of an integer $\mathrm{H}_{t}(n ; k)$ are given by the following.

Proposition 1.2 ([15]). Suppose that there exists an integer $\mathrm{H}_{t}(n ; k)$ for some $n \geq k \geq 3$ and some divisor t of $2 n k$.

(1) If $t$ divides $n k$, then $n k \equiv 0(\bmod 4)$ or $n k \equiv-t \equiv \pm 1(\bmod 4)$.

(2) If $t=2 n k$, then $k$ must be even.

(3) If $t \neq 2 n k$ does not divide $n k$, then $t+2 n k \equiv 0(\bmod 8)$.

We point out that these conditions are not sufficient, in fact in the same paper the authors show that there is no integer $\mathrm{H}_{3 n}(n ; 3)$ and no integer $\mathrm{H}_{8}(4 ; 3)$.

The support of an integer Heffter array $A$, denoted by $\operatorname{supp}(A)$, is defined to be the set of the absolute values of the elements contained in $A$. It is immediate to see that an integer $\mathrm{H}_{2}(n ; k)$ is nothing but an integer $\mathrm{H}_{1}(n ; k)$, since in both cases the support is $\{1,2, \ldots, n k\}$.

In this paper we study the connection between relative Heffter arrays and biembeddings. In particular, in Section 2 we recall well known definitions and results about simple orderings and cycle decompositions. Then, in Section 3 we explain how relative Heffter arrays $\mathrm{H}_{t}(n ; k)$ can be used to construct biembeddings of cyclic $k$-cycle decompositions of the complete multipartite graph $K_{\frac{2 n k+t}{t} \times t}$ into an orientable surface. Direct constructions of globally simple integer $\mathrm{H}_{t}(n ; 3)$ with $t=n, 2 n$ for any odd $n$ and of globally simple integer $\mathrm{H}_{k}(n ; k)$ for $k=7,9$ and $n \equiv 3(\bmod 4)$ are described in Section 4. Combining the results of these sections we prove the following.

Theorem 1.3. There exists a cellular biembedding of a pair of cyclic $k$-cycle decompositions of $K_{\frac{2 n k+t}{t} \times t}$ into an orientable surface in each of the following cases:

(1) $k=3, t \in\{n, 2 n\}$ and $n$ is odd;

(2) $k \in\{3,5,7,9\}, t=k$ and $n \equiv 3(\bmod 4)$.

Finally, in Section 5 we introduce a further generalization, called Archdeacon array, of the classical concept of Heffter array. We show some examples and how both cycle decompositions and biembeddings can be obtained also using these arrays. 


\section{Simple orderings and cycle decompositions}

Given two integers $a \leq b$, we denote by $[a, b]$ the interval containing the integers $a, a+1$, $\ldots, b$. If $a>b$, then $[a, b]$ is empty.

If $A$ is an $m \times n$ p.f. array, the rows and the columns of $A$ will be denoted by $\bar{R}_{1}, \ldots, \bar{R}_{m}$ and by $\bar{C}_{1}, \ldots, \bar{C}_{n}$, respectively. We will denote by $\mathcal{E}(A)$ the unordered list of the elements of the filled cells of $A$. Analogously, by $\mathcal{E}\left(\bar{R}_{i}\right)$ and $\mathcal{E}\left(\bar{C}_{j}\right)$ we mean the unordered lists of elements of the $i$-th row and of the $j$-th column, respectively, of $A$. Also, we define the skeleton of $A$, denoted by $\operatorname{skel}(A)$, to be the set of the filled positions of $A$.

Given a finite subset $T$ of an abelian group $G$ and an ordering $\omega=\left(t_{1}, t_{2}, \ldots, t_{k}\right)$ of the elements in $T$, let $s_{i}=\sum_{j=1}^{i} t_{j}$, for any $i \in[1, k]$, be the $i$-th partial sum of $\omega$ and set $\mathcal{S}(\omega)=\left(s_{1}, \ldots, s_{k}\right)$. The ordering $\omega$ is said to be simple if $s_{b} \neq s_{c}$ for all $1 \leq b<c \leq k$ or, equivalently, if there is no proper subsequence of $\omega$ that sums to 0 . Note that if $\omega$ is a simple ordering so is $\omega^{-1}=\left(t_{k}, t_{k-1}, \ldots, t_{1}\right)$. We point out that there are several interesting problems and conjectures about distinct partial sums: see, for instance, [1, 5, 14, 19, 23]. Given an $m \times n$ p.f. array $A$, by $\omega_{\bar{R}_{i}}$ and $\omega_{\bar{C}_{j}}$ we will denote, respectively, an ordering of $\mathcal{E}\left(\bar{R}_{i}\right)$ and of $\mathcal{E}\left(\bar{C}_{j}\right)$. If for any $i \in[1, m]$ and for any $j \in[1, n]$, the orderings $\omega_{\bar{R}_{i}}$ and $\omega_{\bar{C}_{j}}$ are simple, we define by $\omega_{r}=\omega_{\bar{R}_{1}} \circ \cdots \circ \omega_{\bar{R}_{m}}$ the simple ordering for the rows and by $\omega_{c}=\omega_{\bar{C}_{1}} \circ \cdots \circ \omega_{\bar{C}_{n}}$ the simple ordering for the columns. Moreover, by natural ordering of a row (column) of $A$ we mean the ordering from left to right (from top to bottom). A p.f. array $A$ on an abelian group $G$ is said to be

- simple if each row and each column of $A$ admits a simple ordering;

- globally simple if the natural ordering of each row and each column of $A$ is simple.

Clearly if $k \leq 5$, then every square relative Heffter array is (globally) simple.

We recall some basic definitions about graphs and graph decompositions. Given a graph $\Gamma$, by $V(\Gamma)$ and $E(\Gamma)$ we mean the vertex set and the edge set of $\Gamma$, respectively. We will denote by $K_{v}$ the complete graph of order $v$ and by $K_{q \times r}$ the complete multipartite graph with $q$ parts each of size $r$. Obviously $K_{q \times 1}$ is nothing but the complete graph $K_{q}$. Let $G$ be an additive group (not necessarily abelian) and let $\Lambda \subseteq G \backslash\{0\}$ such that $\Lambda=-\Lambda$, which means that for every $\lambda \in \Lambda$ we have also $-\lambda \in \Lambda$. The Cayley graph on $G$ with connection set $\Lambda$, denoted by Cay $[G: \Lambda]$, is the simple graph having $G$ as vertex set and such that two vertices $x$ and $y$ are adjacent if and only if $x-y \in \Lambda$. Note that, if $\Lambda=G \backslash\{0\}$, the Cayley graph is the complete graph whose vertex set is $G$ and, if $\Lambda=G \backslash J$ for some subgroup $J$ of $G$, the Cayley graph is the complete multipartite graph $K_{q \times r}$ where $q=|G: J|$ and $r=|J|$.

The following are well known definitions and results which can be found, for instance, in [8]. Let $\Gamma$ be a subgraph of a graph $K$. A $\Gamma$-decomposition of $K$ is a set $\mathcal{D}$ of subgraphs of $K$ isomorphic to $\Gamma$ whose edges partition $E(K)$. If the vertices of $K$ belong to a group $G$, given $g \in G$, by $\Gamma+g$ one means the graph whose vertex set is $V(\Gamma)+g$ and whose edge set is $\{\{x+g, y+g\} \mid\{x, y\} \in E(\Gamma)\}$. An automorphism group of a $\Gamma$-decomposition $\mathcal{D}$ of $K$ is a group of bijections on $V(K)$ leaving $\mathcal{D}$ invariant. A $\Gamma$-decomposition of $K$ is said to be regular under a group $G$ or $G$-regular if it admits $G$ as an automorphism group acting sharply transitively on $V(K)$. Here we consider cyclic cycle decompositions, namely decompositions which are regular under a cyclic group and with $\Gamma$ a cycle. Finally, two graph decompositions $\mathcal{D}$ and $\mathcal{D}^{\prime}$ of a simple graph $K$ are said orthogonal if and only if for any $B$ of $\mathcal{D}$ and any $B^{\prime}$ of $\mathcal{D}^{\prime}, B$ intersects $B^{\prime}$ in at most one edge. 
The relationship between simple relative Heffter arrays and cyclic cycle decompositions of the complete multipartite graph is explained in [15]. Here we briefly recall the following result.

Proposition 2.1 ([15, Proposition 2.9]). Let $A$ be a $\mathrm{H}_{t}(m, n ; s, k)$ simple with respect to the orderings $\omega_{r}$ and $\omega_{c}$. Then:

(1) there exists a cyclic s-cycle decomposition $\mathcal{D}_{\omega_{r}}$ of $K_{\frac{2 m s+t}{t} \times t}$;

(2) there exists a cyclic $k$-cycle decomposition $\mathcal{D}_{\omega_{c}}$ of $K_{\frac{2 n k+t}{t} \times t}$;

(3) the cycle decompositions $\mathcal{D}_{\omega_{r}}$ and $\mathcal{D}_{\omega_{c}}$ are orthogonal.

The arrays we are going to construct are square with a diagonal structure, so it is convenient to introduce the following notation. If $A$ is an $n \times n$ array, for $i \in[1, n]$ we define the $i$-th diagonal

$$
D_{i}=\{(i, 1),(i+1,2), \ldots,(i-1, n)\} .
$$

Here all the arithmetic on the row and the column indices is performed modulo $n$, where the set of reduced residues is $\{1,2, \ldots, n\}$. We say that the diagonals $D_{i}, D_{i+1}, \ldots, D_{i+r}$ are consecutive diagonals.

Definition 2.2. Let $k \geq 1$ be an integer. We will say that a square p.f. array $A$ of size $n \geq k$ is

- $k$-diagonal if the non empty cells of $A$ are exactly those of $k$ diagonals;

- cyclically $k$-diagonal if the nonempty cells of $A$ are exactly those of $k$ consecutive diagonals.

Let $A$ be a $k$-diagonal array of size $n>k$. A set $S=\left\{D_{r+1}, D_{r+2}, \ldots, D_{r+\ell}\right\}$ is said to be an empty strip of width $\ell$ if $D_{r+1}, D_{r+2}, \ldots, D_{r+\ell}$ are empty diagonals, while $D_{r}$ and $D_{r+\ell+1}$ are filled diagonals.

Definition 2.3. Let $A$ be a $k$-diagonal array of size $n>k$. We will say that $A$ is a $k$ diagonal array with width $\ell$ if all the empty strips of $A$ have width $\ell$.

An array of this kind will be given in Example 4.9.

\section{Relation with biembeddings}

In [2], Archdeacon introduced Heffter arrays also in view of their applications and, in particular, since they are useful for finding biembeddings of cycle decompositions, as shown, for instance, in $[11,13,16]$. In this section, generalizing some of Archdeacon's results we show how starting from a relative Heffter array it is possible to obtain suitable biembeddings.

We recall the following definition, see [20].

Definition 3.1. An embedding of a graph $\Gamma$ in a surface $\Sigma$ is a continuous injective mapping $\psi: \Gamma \rightarrow \Sigma$, where $\Gamma$ is viewed with the usual topology as 1-dimensional simplicial complex.

The connected components of $\Sigma \backslash \psi(\Gamma)$ are called $\psi$-faces. If each $\psi$-face is homeomorphic to an open disc, then the embedding $\psi$ is said to be cellular. 
Definition 3.2. A biembedding of two cycle decompositions $\mathcal{D}$ and $\mathcal{D}^{\prime}$ of a simple graph $\Gamma$ is a face 2-colorable embedding of $\Gamma$ in which one color class is comprised of the cycles in $\mathcal{D}$ and the other class contains the cycles in $\mathcal{D}^{\prime}$.

Following the notation given in [2], for every edge $e$ of a graph $\Gamma$, let $e^{+}$and $e^{-}$denote its two possible directions and let $\tau$ be the involution swapping $e^{+}$and $e^{-}$for every $e$. Let $D(\Gamma)$ be the set of all directed edges of $\Gamma$ and, for any $v \in V(\Gamma)$, call $D_{v}$ the set of edges directed out of $v$. A local rotation $\rho_{v}$ is a cyclic permutation of $D_{v}$. If we select a local rotation for each vertex of $\Gamma$, then all together they form a rotation of $D(\Gamma)$. We recall the following result, see $[2,18,21]$.

Theorem 3.3. A rotation $\rho$ on $\Gamma$ is equivalent to a cellular embedding of $\Gamma$ in an orientable surface. The face boundaries of the embedding corresponding to $\rho$ are the orbits of $\rho \circ \tau$.

Given a relative Heffter array $A=\mathrm{H}_{t}(m, n ; s, k)$, the orderings $\omega_{r}$ and $\omega_{c}$ are said to be compatible if $\omega_{c} \circ \omega_{r}$ is a cycle of length $|\mathcal{E}(A)|$.

Theorem 3.4. Let $A$ be a relative Heffter array $\mathrm{H}_{t}(m, n ; s, k)$ that is simple with respect to the compatible orderings $\omega_{r}$ and $\omega_{c}$. Then there exists a cellular biembedding of the cyclic cycle decompositions $\mathcal{D}_{\omega_{r}^{-1}}$ and $\mathcal{D}_{\omega_{c}}$ of $K_{\frac{2 n k+t}{t} \times t}$ into an orientable surface of genus

$$
g=1+\frac{(n k-n-m-1)(2 n k+t)}{2} .
$$

Proof. Since the orderings $\omega_{r}$ and $\omega_{c}$ are compatible, we have that $\omega_{c} \circ \omega_{r}$ is a cycle of length $|\mathcal{E}(A)|$. Let us consider the permutation $\bar{\rho}_{0}$ on $\pm \mathcal{E}(A)=\mathbb{Z}_{2 n k+t} \backslash \frac{2 n k+t}{t} \mathbb{Z}_{2 n k+t}$, where $\frac{2 n k+t}{t} \mathbb{Z}_{2 n k+t}$ denotes the subgroup of $\mathbb{Z}_{2 n k+t}$ of order $t$, defined by:

$$
\bar{\rho}_{0}(a)= \begin{cases}-\omega_{r}(a) & \text { if } a \in \mathcal{E}(A) ; \\ \omega_{c}(-a) & \text { if } a \in-\mathcal{E}(A) .\end{cases}
$$

Note that, if $a \in \mathcal{E}(A)$, then $\bar{\rho}_{0}^{2}(a)=\omega_{c} \circ \omega_{r}(a)$ and hence $\bar{\rho}_{0}^{2}$ acts cyclically on $\mathcal{E}(A)$. Also $\bar{\rho}_{0}$ exchanges $\mathcal{E}(A)$ with $-\mathcal{E}(A)$. Thus it acts cyclically on $\pm \mathcal{E}(A)$.

We note that the graph $K_{\frac{2 n k+t}{t} \times t}$ is nothing but Cay $\left[\mathbb{Z}_{2 n k+t}: \mathbb{Z}_{2 n k+t} \backslash \frac{2 n k+t}{t} \mathbb{Z}_{2 n k+t}\right]$ that is Cay $\left[\mathbb{Z}_{2 n k+t}: \pm \mathcal{E}(A)\right]$. Now, we define the map $\rho$ on the set of the oriented edges of the Cayley graph Cay $\left[\mathbb{Z}_{2 n k+t}: \pm \mathcal{E}(A)\right]$ so that:

$$
\rho((x, x+a))=\left(x, x+\bar{\rho}_{0}(a)\right) .
$$

Since $\bar{\rho}_{0}$ acts cyclically on $\pm \mathcal{E}(A)$ the map $\rho$ is a rotation of Cay $\left[\mathbb{Z}_{2 n k+t}: \pm \mathcal{E}(A)\right]$. Hence, by Theorem 3.3, there exists a cellular embedding $\sigma$ of Cay $\left[\mathbb{Z}_{2 n k+t}: \pm \mathcal{E}(A)\right]$ in an orientable surface so that the face boundaries correspond to the orbits of $\rho \circ \tau$ where $\tau((x, x+a))=(x+a, x)$. Let us consider the oriented edge $(x, x+a)$ with $a \in \mathcal{E}(A)$, and let $\bar{C}$ be the column containing $a$. Since $a \in \mathcal{E}(A),-a \in-\mathcal{E}(A)$ and we have that:

$$
\rho \circ \tau((x, x+a))=\rho((x+a,(x+a)-a))=\left(x+a, x+a+\omega_{c}(a)\right) .
$$

Thus $(x, x+a)$ belongs to the boundary of the face $F_{1}$ delimited by the oriented edges:

$$
\begin{aligned}
& (x, x+a),\left(x+a, x+a+\omega_{c}(a)\right), \\
& \quad\left(x+a+\omega_{c}(a), x+a+\omega_{c}(a)+\omega_{c}^{2}(a)\right), \ldots,\left(x+\sum_{i=0}^{|\mathcal{E}(\bar{C})|-2} \omega_{c}^{i}(a), x\right) .
\end{aligned}
$$


We note that the cycle associated to the face $F_{1}$ is:

$$
\left(x, x+a, x+a+\omega_{c}(a), \ldots, x+\sum_{i=0}^{|\mathcal{E}(\bar{C})|-2} \omega_{c}^{i}(a)\right) .
$$

Let us now consider the oriented edge $(x, x+a)$ with $a \notin \mathcal{E}(A)$. Hence $-a \in \mathcal{E}(A)$, and we name by $\bar{R}$ the row containing the element $-a$. Since $-a \in \mathcal{E}(A)$ we have that:

$$
\rho \circ \tau((x, x+a))=\rho((x+a,(x+a)-a))=\left(x+a, x+a-\omega_{r}(-a)\right) .
$$

Thus $(x, x+a)$ belongs to the boundary of the face $F_{2}$ delimited by the oriented edges:

$$
\begin{aligned}
& (x, x+a),\left(x-(-a), x-(-a)-\omega_{r}(-a)\right) \\
& \left(x-(-a)-\omega_{r}(-a), x-(-a)-\omega_{r}(-a)-\omega_{r}^{2}(-a)\right), \ldots,\left(x-\sum_{i=0}^{|\mathcal{E}(\bar{R})|-2} \omega_{r}^{i}(-a), x\right) .
\end{aligned}
$$

Since $A$ is a Heffter array and $\omega_{r}$ acts cyclically on $\mathcal{E}(\bar{R})$, for any $j \in[1,|\mathcal{E}(\bar{R})|]$ we have that:

$$
-\sum_{i=0}^{j-1} \omega_{r}^{i}(-a)=\sum_{i=j}^{|\mathcal{E}(\bar{R})|-1} \omega_{r}^{i}(-a)=\sum_{i=1}^{|\mathcal{E}(\bar{R})|-j} \omega_{r}^{|\mathcal{E}(\bar{R})|-i}(-a)=\sum_{i=1}^{|\mathcal{E}(\bar{R})|-j} \omega_{r}^{-i}(-a) .
$$

It follows that the cycle associated to the face $F_{2}$ can be written also as:

$$
\left(x, x+\sum_{i=1}^{|\mathcal{E}(\bar{R})|-1} \omega_{r}^{-i}(-a), x+\sum_{i=1}^{|\mathcal{E}(\bar{R})|-2} \omega_{r}^{-i}(-a), \ldots, x+\omega_{r}^{-1}(-a)\right) .
$$

Therefore any nonoriented edge $\{x, x+a\}$ belongs to the boundaries of exactly two faces: one of type $F_{1}$ and one of type $F_{2}$. Hence the embedding is 2-colorable.

Moreover, it is easy to see that those face boundaries are the cycles obtained from the relative Heffter array $A$ following the orderings $\omega_{c}$ and $\omega_{r}^{-1}$.

To calculate the genus $g$ it suffices to recall that $V-S+F=2-2 g$, where $V, S$ and $F$ denote the number of vertices, edges and faces determined by the embedding on the surface, respectively. We have $V=2 n k+t, S=n k(2 n k+t)$ and $F=(2 n k+t)(n+m)$.

Looking for compatible orderings in the case of a globally simple Heffter array led us to investigate the following problem introduced in [12]. Let $A$ be an $m \times n$ toroidal p.f. array. By $r_{i}$ we denote the orientation of the $i$-th row, precisely $r_{i}=1$ if it is from left to right and $r_{i}=-1$ if it is from right to left. Analogously, for the $j$-th column, if its orientation $c_{j}$ is from top to bottom then $c_{j}=1$ otherwise $c_{j}=-1$. Assume that an orientation $\mathcal{R}=\left(r_{1}, \ldots, r_{m}\right)$ and $\mathcal{C}=\left(c_{1}, \ldots, c_{n}\right)$ is fixed. Given an initial filled cell $\left(i_{1}, j_{1}\right)$ consider the sequence $L_{\mathcal{R}, \mathcal{C}}\left(i_{1}, j_{1}\right)=\left(\left(i_{1}, j_{1}\right),\left(i_{2}, j_{2}\right), \ldots,\left(i_{\ell}, j_{\ell}\right),\left(i_{\ell+1}, j_{\ell+1}\right), \ldots\right)$ where $j_{\ell+1}$ is the column index of the filled cell $\left(i_{\ell}, j_{\ell+1}\right)$ of the row $\bar{R}_{i_{\ell}}$ next to $\left(i_{\ell}, j_{\ell}\right)$ in the orientation $r_{i_{\ell}}$, and where $i_{\ell+1}$ is the row index of the filled cell of the column $\bar{C}_{j \ell+1}$ next to $\left(i_{\ell}, j_{\ell+1}\right)$ in the orientation $c_{j_{\ell+1}}$. The problem is the following: 
Crazy Knight's Tour Problem. Given a toroidal p.f. array $A$, do there exist $\mathcal{R}$ and $\mathcal{C}$ such that the list $L_{\mathcal{R}, \mathcal{C}}$ covers all the filled cells of $A$ ?

By $P(A)$ we will denote the Crazy Knight's Tour Problem for a given array $A$. Also, given a filled cell $(i, j)$, if $L_{\mathcal{R}, \mathcal{C}}(i, j)$ covers all the filled positions of $A$ we will say that $(\mathcal{R}, \mathcal{C})$ is a solution of $P(A)$. For known results about this problem see [12]. The relationship between the Crazy Knight's Tour Problem and globally simple relative Heffter arrays is explained in the following result which is an easy consequence of Theorem 3.4.

Corollary 3.5. Let $A$ be a globally simple relative Heffter array $\mathrm{H}_{t}(m, n ; s, k)$ such that $P(A)$ admits a solution $(\mathcal{R}, \mathcal{C})$. Then there exists a biembedding of the cyclic cycle decompositions $\mathcal{D}_{\omega_{r}^{-1}}$ and $\mathcal{D}_{\omega_{c}}$ of $K_{\frac{2 n k+t}{t} \times t}$ into an orientable surface.

Extending [11, Theorem 1.1] to the relative case, we have the following result (see also [12, Theorem 2.7]).

Proposition 3.6. If there exist compatible simple orderings $\omega_{r}$ and $\omega_{c}$ for a $\mathrm{H}_{t}(m, n ; s, k)$, then one of the following cases occurs:

(1) $m, n, s, k$ are all odd;

(2) $m$ is odd and $n, k$ are even;

(3) $n$ is odd and $m$, $t$ are even.

Given a positive integer $n$, let $0<\ell_{1}<\ell_{2}<\cdots<\ell_{k}<n$ be integers. We denote by $A_{n}=A_{n}\left(\ell_{1}, \ell_{2}, \ldots, \ell_{k}\right)$ a $k$-diagonal p.f. array of size $n$ whose filled diagonals are $D_{\ell_{1}}, D_{\ell_{2}}, \ldots, D_{\ell_{k}}$. Let $M=\operatorname{lcm}\left(\ell_{2}-\ell_{1}, \ell_{3}-\ell_{2}, \ldots, \ell_{k}-\ell_{k-1}, \ell_{k}-\ell_{1}\right)$ and set $A_{n+M}=$ $A_{n+M}\left(\ell_{1}, \ell_{2}, \ldots, \ell_{k}\right)$. We now study the Crazy Knight's Tour Problem for such arrays $A_{n}$. As a consequence, we will obtain new biembeddings of cycle decompositions of complete graphs on orientable surfaces.

Theorem 3.7. Suppose that the problem $P\left(A_{n}\right)$ admits a solution $(\mathcal{R}, \mathcal{C})$ where $\mathcal{R}=(1,1$, $\ldots, 1)$ and $\mathcal{C}=\left(c_{1}, c_{2}, \ldots, c_{n-\ell_{k}+1}, 1,1, \ldots, 1\right)$. Then $P\left(A_{n+M}\right)$ admits the solution $\left(\mathcal{R}^{\prime}, \mathcal{C}^{\prime}\right)$ where $\mathcal{R}^{\prime}=(1,1, \ldots, 1)$ and $\mathcal{C}^{\prime}=\left(c_{1}, c_{2}, \ldots, c_{n-\ell_{k}+1}, 1,1, \ldots, 1\right)$.

Proof. We denote by $E$ the set of indices $i$ such that $c_{i}=-1$ and by $B_{n}$ the p.f. array of size $n$ obtained from $A_{n}$ by replacing each column $\bar{C}_{j}$, when $j \notin E$, with an empty column. Also, we denote by $B_{n+M}$ the p.f. array of size $n+M$ obtained from $A_{n+M}$ in the same way using the same set $E$. As $E \subseteq\left[1, n-\ell_{k}+1\right]$, the nonempty cells of $B_{n}$ are of the form $\left((e-1)+\ell_{i}, e\right)$ for $e \in E$ and $i \in[1, k]$. Since $(e-1)+\ell_{i} \leq n$, we have $\operatorname{skel}\left(B_{n}\right)=\operatorname{skel}\left(B_{n+M}\right)$. So we can set $B=\operatorname{skel}\left(B_{n}\right)=\operatorname{skel}\left(B_{n+M}\right)$.

For any $x=\left(i_{1}, j_{1}\right) \in B$, consider the sequence $X=L_{\mathcal{R}, \mathcal{C}}\left(i_{1}, j_{1}\right)$ defined on $\operatorname{skel}\left(A_{n}\right)$ and let $y$ be the second element of $X$ that belongs to $B$ if $|X \cap B| \geq 2, y=x$ otherwise. Define $\vartheta_{n}: B \rightarrow B$ by setting $\vartheta_{n}(x)=y$. Take $\left(\mathcal{R}^{\prime}, \mathcal{C}^{\prime}\right)$ as in the statement and define the map $\vartheta_{n+M}: B \rightarrow B$ as before considering the sequence $L_{\mathcal{R}^{\prime}, \mathcal{C}^{\prime}}(x)$ defined on $\operatorname{skel}\left(A_{n+M}\right)$.

In order to prove that $\vartheta_{n}(x)=\vartheta_{n+M}(x)$, for any $h \in[1, k]$, we set:

$$
\sigma(h)=\left\{\begin{array}{ll}
\ell_{1}-\ell_{k-1} & \text { if } h=1 ; \\
\ell_{2}-\ell_{k} & \text { if } h=2 ; \\
\ell_{h}-\ell_{h-2} & \text { otherwise }
\end{array} \quad \text { and } \quad \delta(h)= \begin{cases}\ell_{1}-\ell_{k} & \text { if } h=1 ; \\
\ell_{h}-\ell_{h-1} & \text { otherwise }\end{cases}\right.
$$


Set $x=\left(i_{1}, j_{1}\right) \in B$, hence $x \in D_{\ell_{h}}$ for some $h \in[1, k]$. We have that

$$
\vartheta_{n}(x)=\left(i_{1}+\delta(h) \lambda-\sigma(h), j_{1}+\delta(h) \lambda\right) \quad(\bmod n)
$$

where $\lambda$ is the minimum positive integer such that $\left(j_{1}+\delta(h) \lambda\right)(\bmod n) \in E$. Similarly

$$
\vartheta_{n+M}(x)=\left(i_{1}+\delta(h) \lambda^{\prime}-\sigma(h), j_{1}+\delta(h) \lambda^{\prime}\right) \quad(\bmod n+M)
$$

where $\lambda^{\prime}$ is the minimum positive integer such that $\left(j_{1}+\delta(h) \lambda^{\prime}\right)(\bmod n+M) \in E$. Write $j_{1}+\delta(h) \lambda=q n+r$ where $1 \leq r \leq n$, which means $r \in E$.

If $q=0$, we clearly have $\lambda^{\prime}=\lambda$ and hence $\vartheta_{n+M}(x)=\vartheta_{n}(x)$. Otherwise, since the last $M$ elements of $\mathcal{C}^{\prime}$ are equal to 1 , we have that $\lambda^{\prime}=\lambda+\frac{q M}{\delta(h)}$. Hence:

$$
\begin{aligned}
\vartheta_{n+M}(x) & =\left(i_{1}+\delta(h)\left(\lambda+\frac{q M}{\delta(h)}\right)-\sigma(h), j_{1}+\delta(h)\left(\lambda+\frac{q M}{\delta(h)}\right)\right) \quad(\bmod n+M) \\
& =\left(i_{1}+\delta(h) \lambda+q M-\sigma(h), j_{1}+\delta(h) \lambda+q M\right) \quad(\bmod n+M) \\
& =\left(\left(i_{1}-j_{1}\right)+q(n+M)+r-\sigma(h), q(n+M)+r\right) \quad(\bmod n+M) \\
& =\left(\left(i_{1}-j_{1}\right)+r-\sigma(h), r\right) \quad(\bmod n+M) .
\end{aligned}
$$

Hence

It is not hard to see that $1 \leq\left(i_{1}-j_{1}\right)+r-\sigma(h) \leq n$; also recall that $1 \leq r \leq n$.

$$
\vartheta_{n+M}(x)=\left(\left(i_{1}-j_{1}\right)+r-\sigma(h), r\right) .
$$

On the other hand, by $j_{1}+\delta(h) \lambda=q n+r$, we obtain:

$$
\left(\left(i_{1}-j_{1}\right)+r-\sigma(h), r\right)=\left(i_{1}+\delta(h) \lambda-\sigma(h), j_{1}+\delta(h) \lambda\right) \quad(\bmod n)=\vartheta_{n}(x) .
$$

So we have proved that $\vartheta_{n+M}(x)=\vartheta_{n}(x)$ for any $x \in B$.

For any $(i, j) \in \operatorname{skel}\left(A_{n}\right)$, since $(\mathcal{R}, \mathcal{C})$ is a solution of $P\left(A_{n}\right)$, we have $L_{\mathcal{R}, \mathcal{C}}(i, j) \cap$ $B=B$. Moreover, since $\vartheta_{n}(x)=\vartheta_{n+M}(x)$ for any $x \in B$, it follows that for any $\left(i^{\prime}, j^{\prime}\right) \in \operatorname{skel}\left(A_{n+M}\right)$ we have $L_{\mathcal{R}^{\prime}, \mathcal{C}^{\prime}}\left(i^{\prime}, j^{\prime}\right) \cap B$ is either $B$ or $\emptyset$. If there exists $(\bar{\imath}, \bar{\jmath}) \in$ $\operatorname{skel}\left(A_{n+M}\right)$ such that $L_{\mathcal{R}^{\prime}, \mathcal{C}^{\prime}}(\bar{\imath}, \bar{\jmath}) \cap B=\emptyset$ then for any $\lambda^{\prime} \in \mathbb{N}$, the cell $\left(\bar{\imath}+\delta(\bar{h}) \lambda^{\prime}, \bar{\jmath}+\right.$ $\left.\delta(\bar{h}) \lambda^{\prime}\right)(\bmod n+M)$ is not in $B$. On the other hand there exists $\lambda \in \mathbb{N}$, such that $(\bar{\imath}+\delta(\bar{h}) \lambda, \bar{\jmath}+\delta(\bar{h}) \lambda)(\bmod n) \in B$, since $(\mathcal{R}, \mathcal{C})$ is a solution of $P\left(A_{n}\right)$. Also, since $\delta(\bar{h})$ divides $M$ there exists $\bar{q} \in \mathbb{N}$ such that $(\bar{\imath}+\delta(\bar{h}) \bar{\lambda}, \bar{\jmath}+\delta(\bar{h}) \bar{\lambda})(\bmod n+M) \in B$, where $\bar{\lambda}=\lambda+\bar{q} M / \delta(\bar{h})$. Hence $L_{\mathcal{R}^{\prime}, \mathcal{C}^{\prime}}(\bar{l}, \bar{\jmath}) \cap B \neq \emptyset$, which is a contradiction. Thus it follows that $\left(\mathcal{R}^{\prime}, \mathcal{C}^{\prime}\right)$ is a solution of $P\left(A_{n+M}\right)$.

Corollary 3.8. Let $k \equiv 3(\bmod 4)$ and $n \equiv 1(\bmod 4)$ be such that $n \geq k$ and $3 \leq k \leq$ 119. Let $A_{n}$ be a $k$-diagonal array whose filled diagonals are $D_{1}, D_{2}, \ldots, D_{k-3}, D_{k-1}$, $D_{k}$ and $D_{k+1}$. Then $P\left(A_{n}\right)$ admits a solution.

Proof. Let $k=4 h+3$ and $M=\operatorname{lcm}(2,4 h+3)$, that is $M=2(4 h+3)$. For any $1 \leq h \leq 29$, with the help of a computer, we have checked the existence of a solution of $P\left(A_{n}\right)$ for any $n \in[4 h+5,4 h+5+M]=[4 h+5,12 h+11]$, that satisfies the hypothesis of Theorem 3.7. Hence the claim follows by this theorem.

Corollary 3.9. Let $k \equiv 3(\bmod 4)$ and $n \equiv 1(\bmod 4)$ such that $n \geq k$ and $3 \leq k \leq 119$. Then there exists a globally simple $\mathrm{H}_{1}(n ; k)$ with orderings $\omega_{r}$ and $\omega_{c}$ which are both simple and compatible. As a consequence, there exists a biembedding of cyclic $k$-cycle decompositions of the complete graph $K_{2 n k+1}$ into an orientable surface. 
Proof. The existence of a globally simple $\mathrm{H}_{1}(n ; k)$, whose filled diagonals are $D_{1}, D_{2}, \ldots$, $D_{k-3}, D_{k-1}, D_{k}, D_{k+1}$, was proven in [9]. The result follows from Corollaries 3.5 and 3.8.

\section{Direct constructions of globally simple $\mathrm{H}_{t}(n ; k)$}

Many of the constructions we will present are based on filling in the cells of a set of diagonals. In order to describe these constructions we use the same procedure introduced in [17]. In an $n \times n$ array $A$ the procedure $\operatorname{diag}\left(r, c, s, \Delta_{1}, \Delta_{2}, \ell\right)$ installs the entries

$$
A\left[r+i \Delta_{1}, c+i \Delta_{1}\right]=s+i \Delta_{2} \text { for } i \in[0, \ell-1],
$$

where by $A[i, j]$ we mean the element of $A$ in position $(i, j)$. The parameters used in the diag procedure have the following meaning:

- $r$ denotes the starting row,

- $c$ denotes the starting column,

- $s$ denotes the entry $A[r, c]$,

- $\Delta_{1}$ denotes the increasing value of the row and column at each step,

- $\Delta_{2}$ denotes how much the entry is changed at each step,

- $\ell$ is the length of the chain.

We will write $[a, b]_{(W)}$ to mean $\operatorname{supp}(W)=[a, b]$.

Proposition 4.1. For every odd $n \geq 3$ there exists an integer cyclically 3-diagonal Heffter array $\mathrm{H}_{n}(n ; 3)$.

Proof. We construct an $n \times n$ array $A$ using the following procedures labeled A to E:

$$
\begin{array}{ll}
\mathrm{A}: \operatorname{diag}\left(1,1,-\frac{7 n-9}{2}, 1,7, n\right) ; & \mathrm{B}: \operatorname{diag}\left(1,2, \frac{7 n-3}{2}, 2,-7, \frac{n+1}{2}\right) ; \\
\mathrm{C}: \operatorname{diag}\left(2,3,-5,2,-7, \frac{n-1}{2}\right) ; & \mathrm{D}: \operatorname{diag}\left(2,1, \frac{7 n-13}{2}, 2,-7, \frac{n+1}{2}\right) ; \\
\mathrm{E}: \operatorname{diag}\left(3,2,-10,2,-7, \frac{n-1}{2}\right) . &
\end{array}
$$

We prove that the array constructed above is an integer cyclically 3 -diagonal $\mathrm{H}_{n}(n ; 3)$. To aid in the proof we give a schematic picture of where each of the diagonal procedures fills cells (see Figure 1). Note that each row and each column contain exactly 3 elements. We now check that the elements in every row sum to zero (in $\mathbb{Z}$ ).

Row 1. There is the first value of the $A$ diagonal and of the $B$ diagonal and the last of the $\mathrm{D}$ diagonal. The sum is

$$
-\frac{7 n-9}{2}+\frac{7 n-3}{2}-3=0
$$

Row 2 to $n$. There are two cases depending on whether the row $r$ is even or odd. If $r$ is even, then write $r=2 i+2$ where $i \in\left[0, \frac{n-3}{2}\right]$. Notice that from the $\mathrm{D}, \mathrm{A}$ and $\mathrm{C}$ diagonal cells we get the following sum:

$$
\left(\frac{7 n-13}{2}-7 i\right)+\left(-\frac{7 n-23}{2}+14 i\right)+(-5-7 i)=0 \text {. }
$$




\begin{tabular}{|c|c|c|c|c|c|c|c|c|}
\hline $\mathrm{A}$ & $\mathrm{B}$ & & & & & & & $\mathrm{D}$ \\
\hline $\mathrm{D}$ & $\mathrm{A}$ & $\mathrm{C}$ & & & & & & \\
\hline & $\mathrm{E}$ & $\mathrm{A}$ & $\mathrm{B}$ & & & & & \\
\hline & & $\mathrm{D}$ & $\mathrm{A}$ & $\mathrm{C}$ & & & & \\
\hline & & & $\mathrm{E}$ & $\mathrm{A}$ & $\mathrm{B}$ & & & \\
\hline & & & & $\mathrm{D}$ & $\mathrm{A}$ & $\mathrm{C}$ & & \\
\hline & & & & & $\mathrm{E}$ & $\mathrm{A}$ & $\mathrm{B}$ & \\
\hline & & & & & & $\mathrm{D}$ & $\mathrm{A}$ & $\mathrm{C}$ \\
\hline $\mathrm{B}$ & & & & & & & $\mathrm{E}$ & $\mathrm{A}$ \\
\hline
\end{tabular}

Figure 1: Scheme of construction with $n=9$.

If $r$ is odd, then write $r=2 i+3$ where $i \in\left[0, \frac{n-3}{2}\right]$. From the $\mathrm{E}, \mathrm{A}$ and B diagonal cells we get the following sum:

$$
(-10-7 i)+\left(-\frac{7 n-37}{2}+14 i\right)+\left(\frac{7 n-17}{2}-7 i\right)=0 .
$$

So we have shown that all row sums are zero. Next we check that the columns all add to zero.

Column 1. There is the first value of the A diagonal and of the $D$ diagonal and the last of the $\mathrm{B}$ diagonal. The sum is

$$
-\frac{7 n-9}{2}+\frac{7 n-13}{2}+2=0
$$

Column 2 to $n$. There are two cases depending on whether the column $c$ is even or odd. If $c$ is even, then write $c=2 i+2$ where $i \in\left[0, \frac{n-3}{2}\right]$. Notice that from the $\mathrm{B}, \mathrm{A}$ and E diagonal cells we get the following sum:

$$
\left(\frac{7 n-3}{2}-7 i\right)+\left(-\frac{7 n-23}{2}+14 i\right)+(-10-7 i)=0 .
$$

If $c$ is odd, then write $c=2 i+3$ where $i \in\left[0, \frac{n-3}{2}\right]$. From the C, A and D diagonal cells we get the following sum:

$$
(-5-7 i)+\left(-\frac{7 n-37}{2}+14 i\right)+\left(\frac{7 n-27}{2}-7 i\right)=0 .
$$

So we have shown that each column sums to zero. Also, it is not hard to see that:

$$
\begin{aligned}
& \operatorname{supp}(\mathrm{A})=\left\{1,8,15, \ldots, \frac{7 n-5}{2}\right\} \cup\left\{6,13,20, \ldots, \frac{7 n-9}{2}\right\}, \\
& \operatorname{supp}(\mathrm{B})=\left\{2,9,16, \ldots, \frac{7 n-3}{2}\right\}, \\
& \operatorname{supp}(\mathrm{C})=\left\{5,12,19, \ldots, \frac{7 n-11}{2}\right\}, \\
& \operatorname{supp}(\mathrm{D})=\{3\} \cup\left\{4,11,18, \ldots, \frac{7 n-13}{2}\right\}, \\
& \operatorname{supp}(\mathrm{E})=\left\{10,17,24, \ldots, \frac{7 n-1}{2}\right\},
\end{aligned}
$$

hence $\operatorname{supp}(A)=\left[1, \frac{7 n-1}{2}\right] \backslash\left\{7,14,21, \ldots, \frac{7 n-7}{2}\right\}$. This concludes the proof. 
Example 4.2. Following the proof of Proposition 4.1 we obtain the integer $\mathrm{H}_{9}(9 ; 3)$ below.

\begin{tabular}{|r|r|r|r|r|r|r|r|r|}
\hline-27 & 30 & & & & & & & -3 \\
\hline 25 & -20 & -5 & & & & & & \\
\hline & -10 & -13 & 23 & & & & & \\
\hline & & 18 & -6 & -12 & & & & \\
\hline & & & -17 & 1 & 16 & & & \\
\hline & & & & 11 & 8 & -19 & & \\
\hline & & & & & -24 & 15 & 9 & \\
\hline & & & & & & 4 & 22 & -26 \\
\hline 2 & & & & & & & -31 & 29 \\
\hline
\end{tabular}

We can use this example to briefly explain how the construction has been obtained (a similar idea will be used also in Proposition 4.3 below). First of all, we have to avoid the multiples of $\frac{2 n k}{t}+1=7$, so we work modulo 7 . The diagonal $D_{1}$ consists of elements, all congruent to 1 modulo 7 , arranged in arithmetic progression where, for instance, the central cell is filled with 1 . The other two filled diagonals are obtained in such a way that the elements of $D_{9}$ are all congruent to 2 modulo 7 and the elements of $D_{2}$ are all congruent to -3 modulo 7 . This can be achieved filling the cell $(9,1)$ with the integer 2 : it is now easy to obtain the elements in the remaining cells, remembering that the row/column sums are 0 .

Proposition 4.3. For every odd $n \geq 3$ there exists an integer cyclically 3-diagonal Heffter array $\mathrm{H}_{2 n}(n ; 3)$.

Proof. We construct an $n \times n$ array $A$ using the following procedures labeled A to $\mathrm{E}$ :

$$
\begin{array}{ll}
\mathrm{A}: \operatorname{diag}(1,1,-(4 n-5), 1,8, n) ; & \mathrm{B}: \operatorname{diag}\left(1,2,4 n-2,2,-8, \frac{n+1}{2}\right) ; \\
\mathrm{C}: \operatorname{diag}\left(2,3,-6,2,-8, \frac{n-1}{2}\right) ; & \mathrm{D}: \operatorname{diag}\left(2,1,4 n-7,2,-8, \frac{n+1}{2}\right) ; \\
\mathrm{E}: \operatorname{diag}\left(3,2,-11,2,-8, \frac{n-1}{2}\right) . &
\end{array}
$$

We prove that the array constructed above is an integer cyclically 3 -diagonal $\mathrm{H}_{2 n}(n ; 3)$. To aid in the proof we give a schematic picture of where each of the diagonal procedures fills cells (see Figure 1). Note that each row and each column contain exactly 3 elements. We now check that the elements in every row sum to zero (in $\mathbb{Z}$ ).

Row 1. There is the first value of the $A$ diagonal and of the $B$ diagonal and the last of the $\mathrm{D}$ diagonal. The sum is

$$
-(4 n-5)+(4 n-2)-3=0 .
$$

Row 2 to $n$. There are two cases depending on whether the row $r$ is even or odd. If $r$ is even, then write $r=2 i+2$ where $i \in\left[0, \frac{n-3}{2}\right]$. Notice that from the $\mathrm{D}, \mathrm{A}$ and $\mathrm{C}$ diagonal cells we get the following sum:

$$
(4 n-7-8 i)+(-4 n+13+16 i)+(-6-8 i)=0 .
$$

If $r$ is odd, then write $r=2 i+3$ where $i \in\left[0, \frac{n-3}{2}\right]$. From the $\mathrm{E}, \mathrm{A}$ and B diagonal cells we get the following sum:

$$
(-11-8 i)+(-4 n+21+16 i)+(4 n-10-8 i)=0 .
$$


So we have shown that all row sums are zero. Next we check that the columns all add to zero.

Column 1. There is the first value of the A diagonal and of the $D$ diagonal and the last of the $\mathrm{B}$ diagonal. The sum is

$$
-(4 n-5)+(4 n-7)+2=0 .
$$

Column 2 to $n$. There are two cases depending on whether the column $c$ is even or odd. If $c$ is even, then write $c=2 i+2$ where $i \in\left[0, \frac{n-3}{2}\right]$. Notice that from the $\mathrm{B}, \mathrm{A}$ and $\mathrm{E}$ diagonal cells we get the following sum:

$$
(4 n-2-8 i)+(-4 n+13+16 i)+(-11-8 i)=0 .
$$

If $c$ is odd, then write $c=2 i+3$ where $i \in\left[0, \frac{n-3}{2}\right]$. From the C, A and D diagonal cells we get the following sum:

$$
(-6-8 i)+(-4 n+21+16 i)+(4 n-15-8 i)=0 .
$$

So we have shown that each column sums to zero. Also, it is not hard to see that:

$$
\begin{aligned}
& \operatorname{supp}(\mathrm{A})=\{1,9,17, \ldots, 4 n-3\} \cup\{7,15,23, \ldots, 4 n-5\}, \\
& \operatorname{supp}(\mathrm{B})=\{2,10,18, \ldots, 4 n-2\} \\
& \operatorname{supp}(\mathrm{C})=\{6,14,22, \ldots, 4 n-6\} \\
& \operatorname{supp}(\mathrm{D})=\{3\} \cup\{5,13,21, \ldots, 4 n-7\}, \\
& \operatorname{supp}(\mathrm{E})=\{11,19,27, \ldots, 4 n-1\},
\end{aligned}
$$

hence $\operatorname{supp}(A)=[1,4 n-1] \backslash\{4,8,12, \ldots, 4 n-4\}$. This concludes the proof.

Example 4.4. Following the proof of Proposition 4.3 we obtain the integer $\mathrm{H}_{18}(9 ; 3)$ below.

\begin{tabular}{|r|r|r|r|r|r|r|r|r|}
\hline-31 & 34 & & & & & & & -3 \\
\hline 29 & -23 & -6 & & & & & & \\
\hline & -11 & -15 & 26 & & & & & \\
\hline & & 21 & -7 & -14 & & & & \\
\hline & & & -19 & 1 & 18 & & & \\
\hline & & & & 13 & 9 & -22 & & \\
\hline & & & & & -27 & 17 & 10 & \\
\hline & & & & & & 5 & 25 & -30 \\
\hline 2 & & & & & & & -35 & 33 \\
\hline
\end{tabular}

In the following propositions, since $k>5$, in order to prove that the relative Heffter array $\mathrm{H}_{k}(n ; k)$ constructed is globally simple we have to show that the partial sums of each row and of each column are distinct modulo $2 n k+k$. From now on, the sets $\mathcal{E}\left(\bar{R}_{i}\right)$ and $\mathcal{E}\left(\bar{C}_{i}\right)$ are considered ordered with respect to the natural ordering. Also, by $\mathcal{S}\left(\bar{R}_{i}\right)$ and $\mathcal{S}\left(\bar{C}_{i}\right)$ we will denote the sequence of the partial sums of $\mathcal{E}\left(\bar{R}_{i}\right)$ and $\mathcal{E}\left(\bar{C}_{i}\right)$, respectively. In order to check that the partial sums are distinct the following remark allows to reduce the computations. 
Remark 4.5. Let $A$ be a $\mathrm{H}_{t}(n ; k)$. By the definition of a (relative) Heffter array it easily follows that the $i$-th partial sum $s_{i}$ of a row (or a column) is different from the partial sums $s_{i-2}, s_{i-1}, s_{i+1}$ and $s_{i+2}$ of the same row (column).

Proposition 4.6. For every $n \geq 7$ with $n \equiv 3(\bmod 4)$ there exists an integer cyclically 7-diagonal globally simple $\mathrm{H}_{7}(n ; 7)$.

Proof. We construct an $n \times n$ array $A$ using the following procedures labeled A to N:
A: $\operatorname{diag}\left(3,3,-\frac{n+1}{2}, 2,-1, \frac{n-1}{2}\right)$;
B: $\operatorname{diag}\left(4,4,1,2,1, \frac{n-3}{2}\right)$;
C: $\operatorname{diag}(n-2, n-1,-(5 n+3), 2,-1, n)$;
D: $\operatorname{diag}(2,1,-(4 n+3), 2,-1, n)$;
$\mathrm{E}: \operatorname{diag}\left(1,3, \frac{7 n+3}{4}, 4,1, \frac{n+1}{4}\right)$;
$\mathrm{F}: \operatorname{diag}\left(2,4, \frac{3 n+1}{2}, 4,-1, \frac{n+1}{4}\right)$;
$\mathrm{G}: \operatorname{diag}\left(3,5, \frac{11 n+7}{4}, 4,1, \frac{n+1}{4}\right)$;
$\mathrm{H}: \operatorname{diag}\left(4,6, \frac{5 n+1}{2}, 4,-1, \frac{n-3}{4}\right)$;
I : $\operatorname{diag}\left(3,1,-\frac{9 n+5}{4}, 4,1, \frac{n+1}{4}\right)$;
$\mathrm{J}: \operatorname{diag}\left(4,2,-\frac{5 n+3}{2}, 4,-1, \frac{n+1}{4}\right)$;
$\mathrm{K}: \operatorname{diag}\left(5,3,-\frac{5 n+1}{4}, 4,1, \frac{n+1}{4}\right)$;
$\mathrm{L}: \operatorname{diag}\left(6,4,-\frac{3 n+3}{2}, 4,-1, \frac{n-3}{4}\right)$;
$\mathrm{M}: \operatorname{diag}(n-2,1,6 n+4,2,1, n)$;
$\mathrm{N}: \operatorname{diag}(2, n-1,3 n+2,2,1, n)$.

We also fill the following cells in an ad hoc manner:

$$
A[1,1]=n, \quad A[2,2]=-\frac{n-1}{2} .
$$

We prove that the array constructed above is an integer cyclically 7 -diagonal globally simple $\mathrm{H}_{7}(n ; 7)$. To aid in the proof we give a schematic picture of where each of the diagonal procedures fills cells (see Figure 2). We have placed an X in the ad hoc cells. Note that each row and each column contains exactly 7 elements. We now list the elements and the partial sums of each row. We leave to the reader the direct check that the partial sums are distinct modulo $14 n+7$; for a quicker check keep in mind Remark 4.5.

\begin{tabular}{|c|c|c|c|c|c|c|c|c|c|c|}
\hline X & C & E & M & & & & & N & J & D \\
\hline D & X & C & F & M & & & & & N & K \\
\hline I & D & A & C & G & M & & & & & N \\
\hline N & J & D & B & C & H & M & & & & \\
\hline & N & K & D & A & C & E & M & & & \\
\hline & & N & L & D & B & C & F & M & & \\
\hline & & & N & I & D & A & C & G & M & \\
\hline & & & & N & J & D & B & C & H & M \\
\hline M & & & & & N & K & D & A & C & E \\
\hline F & M & & & & & N & L & D & B & C \\
\hline C & G & M & & & & & N & I & D & A \\
\hline
\end{tabular}

Figure 2: Scheme of construction with $n=11$.

Row 1. There is an ad hoc element, the $\left(\frac{n+5}{2}\right)^{\text {th }}$ value of the $\mathrm{C}$ diagonal, the first one of the E diagonal, the $\left(\frac{n+5}{2}\right)^{\text {th }}$ value of the $\mathrm{M}$ diagonal, the $\left(\frac{n+1}{2}\right)^{\text {th }}$ value of the $\mathrm{N}$ diagonal, the last value of the $\mathrm{J}$ diagonal and the $\left(\frac{n+1}{2}\right)^{\text {th }}$ value of the D diagonal. Namely,

$$
\mathcal{E}\left(\bar{R}_{1}\right)=\left(n,-\frac{11 n+9}{2}, \frac{7 n+3}{4}, \frac{13 n+11}{2}, \frac{7 n+3}{2},-\frac{11 n+3}{4},-\frac{9 n+5}{2}\right)
$$


and

$$
\mathcal{S}\left(\bar{R}_{1}\right)=\left(n,-\frac{9 n+9}{2},-\frac{11 n+15}{4}, \frac{15 n+7}{4}, \frac{29 n+13}{4}, \frac{9 n+5}{2}, 0\right) .
$$

Row 2. There is the first value of the D diagonal, an ad hoc element, the third value of the $\mathrm{C}$ diagonal, the first value of the $\mathrm{F}$ diagonal, the third value of the $\mathrm{M}$ diagonal, the first value of the $\mathrm{N}$ diagonal and the last value of the $\mathrm{K}$ diagonal. Hence

$$
\mathcal{E}\left(\bar{R}_{2}\right)=\left(-(4 n+3),-\frac{n-1}{2},-(5 n+5), \frac{3 n+1}{2}, 6 n+6,3 n+2,-(n+1)\right)
$$

and

$$
\mathcal{S}\left(\bar{R}_{2}\right)=\left(-(4 n+3),-\frac{9 n+5}{2},-\frac{19 n+15}{2},-(8 n+7),-(2 n+1), n+1,0\right) .
$$

Row 3 to $n$. There are four cases depending on the congruence class of $r$ modulo 4 . If $r \equiv 3(\bmod 4)$, then write $r=4 i+3$ where $i \in\left[0, \frac{n-3}{4}\right]$. It is not hard to see that from the N, I, D, A, C, G and M diagonal cells we get:

$$
\begin{aligned}
\mathcal{E}\left(\bar{R}_{4 i+3}\right)= & \frac{7 n+5}{2}+2 i,-\frac{9 n+5}{4}+i,-\frac{9 n+7}{2}-2 i,-\frac{n+1}{2}-2 i \\
& \left.-\frac{11 n+11}{2}-2 i+\varepsilon, \frac{11 n+7}{4}+i, \frac{13 n+13}{2}+2 i-\varepsilon\right),
\end{aligned}
$$

where $\varepsilon=0$ for $i \in\left[0, \frac{n-7}{4}\right]$ while $\varepsilon=n$ for $i=\frac{n-3}{4}$, and

$$
\begin{aligned}
\mathcal{S}\left(\bar{R}_{4 i+3}\right)= & \frac{7 n+5}{2}+2 i, \frac{5 n+5}{4}+3 i,-\frac{13 n+9}{4}+i, \\
& \left.\quad-\frac{15 n+11}{4}-i,-\frac{37 n+33}{4}-3 i+\varepsilon,-\frac{13 n+13}{2}-2 i+\varepsilon, 0\right) .
\end{aligned}
$$

If $r \equiv 0(\bmod 4)$, then write $r=4 i+4$ where $i \in\left[0, \frac{n-7}{4}\right]$. It is not hard to see that from the N, J, D, B, C, H and M diagonal cells we get:

$$
\begin{aligned}
\mathcal{E}\left(\bar{R}_{4 i+4}\right)= & \left(3 n+3+2 i,-\frac{5 n+3}{2}-i,-(4 n+4+2 i),\right. \\
& \left.1+2 i,-(5 n+6+2 i), \frac{5 n+1}{2}-i, 6 n+7+2 i\right)
\end{aligned}
$$

and

$$
\begin{aligned}
\mathcal{S}\left(\bar{R}_{4 i+4}\right)=( & 3 n+3+2 i, \frac{n+3}{2}+i,-\frac{7 n+5}{2}-i \\
& \left.-\frac{7 n+3}{2}+i,-\frac{17 n+15}{2}-i,-(6 n+7+2 i), 0\right) .
\end{aligned}
$$


If $r \equiv 1(\bmod 4)$, then write $r=4 i+5$ where $i \in\left[0, \frac{n-7}{4}\right]$. It is not hard to see that from the N, K, D, A, C, E and M diagonal cells we get:

$$
\begin{aligned}
\mathcal{E}\left(\bar{R}_{4 i+5}\right)= & \frac{7 n+7}{2}+2 i,-\frac{5 n+1}{4}+i,-\frac{9 n+9}{2}-2 i,-\frac{n+3}{2}-2 i, \\
& \left.-\frac{11 n+13}{2}-2 i+\varepsilon, \frac{7 n+7}{4}+i, \frac{13 n+15}{2}+2 i-\varepsilon\right),
\end{aligned}
$$

where $\varepsilon=0$ for $i \in\left[0, \frac{n-11}{4}\right]$ while $\varepsilon=n$ for $i=\frac{n-7}{4}$, and

$$
\begin{aligned}
\mathcal{S}\left(\bar{R}_{4 i+5}\right)= & \frac{7 n+7}{2}+2 i, \frac{9 n+13}{4}+3 i,-\frac{9 n+5}{4}+i \\
& \left.-\frac{11 n+11}{4}-i,-\frac{33 n+37}{4}-3 i+\varepsilon,-\frac{13 n+15}{2}-2 i+\varepsilon, 0\right) .
\end{aligned}
$$

If $r \equiv 2(\bmod 4)$, then write $r=4 i+6$ where $i \in\left[0, \frac{n-7}{4}\right]$. It is not hard to see that from the N, L, D, B, C, F and M diagonal cells we get:

$$
\begin{aligned}
\mathcal{E}\left(\bar{R}_{4 i+6}\right)= & \left(3 n+4+2 i,-\frac{3 n+3}{2}-i,-(4 n+5+2 i),\right. \\
& \left.2+2 i,-(5 n+7+2 i), \frac{3 n-1}{2}-i, 6 n+8+2 i\right)
\end{aligned}
$$

and

$$
\begin{aligned}
\mathcal{S}\left(\bar{R}_{4 i+6}\right)= & \left(3 n+4+2 i, \frac{3 n+5}{2}+i,-\frac{5 n+5}{2}-i,\right. \\
& \left.-\frac{5 n+1}{2}+i,-\frac{15 n+15}{2}-i,-(6 n+8+2 i), 0\right) .
\end{aligned}
$$

Now we list the elements and the partial sums of the columns.

Column 1. There is an ad hoc element, the first value of the $D$ diagonal and of the I diagonal, the second value of the $\mathrm{N}$ diagonal, the first value of the $\mathrm{M}$ diagonal, the last value of the $\mathrm{F}$ diagonal and the second value of the $\mathrm{C}$ diagonal. Namely,

$$
\mathcal{E}\left(\bar{C}_{1}\right)=\left(n,-(4 n+3),-\frac{9 n+5}{4}, 3 n+3,6 n+4, \frac{5 n+5}{4},-(5 n+4)\right)
$$

and

$$
\mathcal{S}\left(\bar{C}_{1}\right)=\left(n,-(3 n+3),-\frac{21 n+17}{4},-\frac{9 n+5}{4}, \frac{15 n+11}{4}, 5 n+4,0\right) .
$$

Column 2. There is the $\left(\frac{n+5}{2}\right)^{\text {th }}$ value of the $C$ diagonal, an ad hoc element, the $\left(\frac{n+3}{2}\right)^{\text {th }}$ value of the $\mathrm{D}$ diagonal, the first value of the $\mathrm{J}$ diagonal, the $\left(\frac{n+5}{2}\right)^{\text {th }}$ value of the $\mathrm{N}$ diagonal and of the M diagonal and the last value of the G diagonal. Namely,

$$
\mathcal{E}\left(\bar{C}_{2}\right)=\left(-\frac{11 n+9}{2},-\frac{n-1}{2},-\frac{9 n+7}{2},-\frac{5 n+3}{2}, \frac{7 n+7}{2}, \frac{13 n+9}{2}, 3 n+1\right)
$$


and

$$
\begin{aligned}
\mathcal{S}\left(\bar{C}_{2}\right)=( & -\frac{11 n+9}{2},-(6 n+4),-\frac{21 n+15}{2}, \\
& \left.-(13 n+9),-\frac{19 n+11}{2},-(3 n+1), 0\right) .
\end{aligned}
$$

Column 3 to $n$. There are four cases depending on the congruence class of $c$ modulo 4 . If $c \equiv 3(\bmod 4)$, then write $c=4 i+3$ where $i \in\left[0, \frac{n-3}{4}\right]$. It is not hard to see that from the M, E, C, A, D, K and $\mathrm{N}$ diagonal cells we get:

$$
\begin{aligned}
\mathcal{E}\left(\bar{C}_{4 i+3}\right)=( & 6 n+5+2 i, \frac{7 n+3}{4}+i,-(5 n+5+2 i), \\
& \left.\quad-\frac{n+1}{2}-2 i,-(4 n+4+2 i),-\frac{5 n+1}{4}+i, 3 n+4+2 i\right)
\end{aligned}
$$

and

$$
\begin{aligned}
\mathcal{S}\left(\bar{C}_{4 i+3}\right)= & \left(6 n+5+2 i, \frac{31 n+23}{4}+3 i, \frac{11 n+3}{4}+i,\right. \\
& \left.\frac{9 n+1}{4}-i,-\frac{7 n+15}{4}-3 i,-(3 n+4+2 i), 0\right) .
\end{aligned}
$$

If $c \equiv 0(\bmod 4)$, then write $c=4 i+4$ where $i \in\left[0, \frac{n-7}{4}\right]$. It is not hard to see that from the $\mathrm{M}, \mathrm{F}, \mathrm{C}, \mathrm{B}, \mathrm{D}, \mathrm{L}$ and $\mathrm{N}$ diagonal cells we get:

$$
\begin{aligned}
\mathcal{E}\left(\bar{C}_{4 i+4}\right)= & \left(\frac{13 n+11}{2}+2 i, \frac{3 n+1}{2}-i,-\frac{11 n+11}{2}-2 i,\right. \\
& \left.1+2 i,-\frac{9 n+9}{2}-2 i,-\frac{3 n+3}{2}-i, \frac{7 n+9}{2}+2 i\right)
\end{aligned}
$$

and

$$
\begin{aligned}
\mathcal{S}\left(\bar{C}_{4 i+4}\right)= & \left(\frac{13 n+11}{2}+2 i, 8 n+6+i, \frac{5 n+1}{2}-i,\right. \\
& \left.\frac{5 n+3}{2}+i,-(2 n+3+i),-\frac{7 n+9}{2}-2 i, 0\right) .
\end{aligned}
$$

If $c \equiv 1(\bmod 4)$, then write $c=4 i+5$ where $i \in\left[0, \frac{n-7}{4}\right]$. It is not hard to see that from the M, G, C, A, D, I and $\mathrm{N}$ diagonal cells we get:

$$
\begin{aligned}
\mathcal{E}\left(\bar{C}_{4 i+5}\right)= & \left(6 n+6+2 i, \frac{11 n+7}{4}+i,-(5 n+6+2 i),\right. \\
& \left.\quad-\frac{n+3}{2}-2 i,-(4 n+5+2 i),-\frac{9 n+1}{4}+i, 3 n+5+2 i\right)
\end{aligned}
$$

and

$$
\begin{aligned}
\mathcal{S}\left(\bar{C}_{4 i+5}\right)= & \left(6 n+6+2 i, \frac{35 n+31}{4}+3 i, \frac{15 n+7}{4}+i\right. \\
& \left.\frac{13 n+1}{4}-i,-\frac{3 n+19}{4}-3 i,-(3 n+5+2 i), 0\right) .
\end{aligned}
$$


If $c \equiv 2(\bmod 4)$, then write $c=4 i+6$ where $i \in\left[0, \frac{n-7}{4}\right]$. It is not hard to see that from the $\mathrm{M}, \mathrm{H}, \mathrm{C}, \mathrm{B}, \mathrm{D}, \mathrm{J}$ and $\mathrm{N}$ diagonal cells we get:

$$
\begin{aligned}
\mathcal{E}\left(\bar{C}_{4 i+6}\right)= & \left(\frac{13 n+13}{2}+2 i, \frac{5 n+1}{2}-i,-\frac{11 n+13}{2}-2 i+\varepsilon,\right. \\
& \left.2+2 i,-\frac{9 n+11}{2}-2 i,-\frac{5 n+5}{2}-i, \frac{7 n+11}{2}+2 i-\varepsilon\right),
\end{aligned}
$$

where $\varepsilon=0$ for $i \in\left[0, \frac{n-11}{4}\right]$ while $\varepsilon=n$ for $i=\frac{n-7}{4}$, and

$$
\begin{aligned}
\mathcal{S}\left(\bar{C}_{4 i+6}\right)= & \left(\frac{13 n+13}{2}+2 i, 9 n+7+i, \frac{7 n+1}{2}-i+\varepsilon,\right. \\
& \left.\frac{7 n+5}{2}+i+\varepsilon,-(n+3+i)+\varepsilon,-\frac{7 n+11}{2}-2 i+\varepsilon, 0\right) .
\end{aligned}
$$

Finally we consider the support of $A$ :

$$
\begin{aligned}
\operatorname{supp}(A) & =\left[1, \frac{n-3}{2}\right]_{(\mathrm{B})} \cup\left\{\frac{n-1}{2}\right\} \cup\left[\frac{n+1}{2}, n-1\right]_{(\mathrm{A})} \cup\{n\} \\
& \cup\left[n+1, \frac{5 n+1}{4}\right]_{(\mathrm{K})} \cup\left[\frac{5 n+5}{4}, \frac{3 n+1}{2}\right]_{(\mathrm{F})} \cup\left[\frac{3 n+3}{2}, \frac{7 n-1}{4}\right]_{(\mathrm{I})} \\
& \cup\left[\frac{7 n+3}{4}, 2 n\right]_{(\mathrm{E})} \cup\left[2 n+2, \frac{9 n+5}{4}\right]_{(\mathrm{I})} \cup\left[\frac{9 n+9}{4}, \frac{5 n+1}{2}\right]_{(\mathrm{H})} \\
& \cup\left[\frac{5 n+3}{2}, \frac{11 n+3}{4}\right]_{(\mathrm{J})} \cup\left[\frac{11 n+7}{4}, 3 n+1\right]_{(\mathrm{G})} \cup[3 n+2,4 n+1]_{(\mathrm{N})} \\
& \cup[4 n+3,5 n+2]_{(\mathrm{D})} \cup[5 n+3,6 n+2]_{(\mathrm{C})} \cup[6 n+4,7 n+3]_{(\mathrm{M})} \\
& =[1,7 n+3] \backslash\{2 n+1,4 n+2,6 n+3\} .
\end{aligned}
$$

This concludes the proof.

Example 4.7. Following the proof of Proposition 4.6 we obtain the integer globally simple $\mathrm{H}_{7}(11 ; 7)$ below.

\begin{tabular}{|r|r|r|r|r|r|r|r|r|r|r|}
\hline 11 & -65 & 20 & 77 & & & & & 40 & -31 & -52 \\
\hline-47 & -5 & -60 & 17 & 72 & & & & & 35 & -12 \\
\hline-26 & -53 & -6 & -66 & 32 & 78 & & & & & 41 \\
\hline 36 & -29 & -48 & 1 & -61 & 28 & 73 & & & & \\
\hline & 42 & -14 & -54 & -7 & -67 & 21 & 79 & & & \\
\hline & & 37 & -18 & -49 & 2 & -62 & 16 & 74 & & \\
\hline & & & 43 & -25 & -55 & -8 & -68 & 33 & 80 & \\
\hline & & & & 38 & -30 & -50 & 3 & -63 & 27 & 75 \\
\hline 70 & & & & & 44 & -13 & -56 & -9 & -58 & 22 \\
\hline 15 & 76 & & & & & 39 & -19 & -51 & 4 & -64 \\
\hline-59 & 34 & 71 & & & & & 45 & -24 & -57 & -10 \\
\hline
\end{tabular}

Proposition 4.8. For every $n \geq 11$ with $n \equiv 3(\bmod 4)$ there exists an integer 9 -diagonal globally simple $\mathrm{H}_{9}(n ; 9)$ with width $\frac{n-9}{2}$. 
Proof. We construct an $n \times n$ array $A$ using the following procedures labeled A to R:
A: $\operatorname{diag}(3,1,5 n+3,1,1, n)$;
B: $\operatorname{diag}(4,1,-(6 n+4), 1,-1, n)$;
C: $\operatorname{diag}(3,6,-(7 n+4), 1,-1, n)$;
D: $\operatorname{diag}(4,6,8 n+5,1,1, n)$;
$\mathrm{E}: \operatorname{diag}\left(1, \frac{n+3}{2},-(2 n), 1,2, \frac{n-1}{2}\right)$;
$\mathrm{F}: \operatorname{diag}\left(\frac{n+3}{2}, 1,2 n+2,1,2, \frac{n-1}{2}\right)$;
$\mathrm{G}: \operatorname{diag}\left(2,2,-(n-2), 1,1, \frac{n-3}{2}\right)$;
$\mathrm{H}: \operatorname{diag}\left(\frac{n+3}{2}, 2,-(2 n+3), 1,-2, \frac{n-3}{2}\right)$;
I : $\operatorname{diag}\left(2, \frac{n+3}{2}, 2 n-1,1,-2, \frac{n-3}{2}\right)$;
$\mathrm{J}: \operatorname{diag}\left(\frac{n+3}{2}, \frac{n+3}{2}, \frac{n-3}{2}, 1,-1, \frac{n-5}{2}\right)$;
$\mathrm{K}: \operatorname{diag}\left(2,1,-(3 n+4), 2,-1, \frac{n+1}{4}\right)$;
L : $\operatorname{diag}\left(1,2,5 n, 2,-1, \frac{n+1}{4}\right)$;
$\mathrm{M}: \operatorname{diag}\left(3,2,-(4 n+3), 2,-1, \frac{n-3}{4}\right)$;
$\mathrm{N}: \operatorname{diag}\left(2,3,4 n+1,2,-1, \frac{n-3}{4}\right)$;
○: $\operatorname{diag}\left(\frac{n+1}{2}, \frac{n+3}{2}, \frac{17 n+9}{4}, 2,1, \frac{n-3}{4}\right)$;
$\mathrm{P}: \operatorname{diag}\left(\frac{n+3}{2}, \frac{n+1}{2},-\frac{15 n+7}{4}, 2,1, \frac{n-3}{4}\right)$;
Q: $\operatorname{diag}\left(\frac{n+3}{2}, \frac{n+5}{2}, \frac{13 n+17}{4}, 2,1, \frac{n-3}{4}\right)$;
$\mathrm{R}: \operatorname{diag}\left(\frac{n+5}{2}, \frac{n+3}{2},-\frac{19 n-1}{4}, 2,1, \frac{n-3}{4}\right)$.

We also fill the following cells in an ad hoc manner:

$$
\begin{array}{lll}
A[1,1]=n-1, & A\left[1, \frac{n+1}{2}\right]=n+2, & A[1, n]=-(5 n+1), \\
A\left[\frac{n+1}{2}, 1\right]=-(3 n), & A\left[\frac{n+1}{2}, \frac{n+1}{2}\right]=n, & A\left[\frac{n+1}{2}, n\right]=n+1, \\
A[n-1, n-1]=-\frac{n-1}{2}, & A[n-1, n]=5 n+2, & A[n, 1]=3 n+3, \\
A\left[n, \frac{n+1}{2}\right]=-(3 n+1), & A[n, n-1]=-(3 n+2), & A[n, n]=1 .
\end{array}
$$

We prove that the array constructed above is an integer 9-diagonal globally simple $\mathrm{H}_{9}(n ; 9)$ with width $\frac{n-9}{2}$. To aid in the proof we give a schematic picture of where each of the diagonal procedures fills cells (see Figure 3). We have placed an X in the ad hoc cells. Note that each row and each column contains exactly 9 elements. Since the filled diagonals are $D_{1}, D_{2}, D_{3}, D_{4}, D_{\frac{n+1}{2}}, D_{\frac{n+3}{2}}, D_{n-2}, D_{n-1}$ and $D_{n}, A$ has two empty strips of size $\frac{n-9}{2}$. We now list the elements and the partial sums of every row. We leave to the reader the direct check that the partial sums are distinct modulo $18 n+9$; for a quicker check keep in mind Remark 4.5.

\begin{tabular}{|c|c|c|c|c|c|c|c|c|c|c|c|c|c|c|}
\hline X & L & D & C & & & & X & E & & & & B & A & X \\
\hline K & G & N & D & C & & & & I & E & & & & B & A \\
\hline A & M & G & L & D & C & & & & I & E & & & & B \\
\hline B & A & K & G & N & D & C & & & & I & E & & & \\
\hline & B & A & M & G & L & D & C & & & & I & E & & \\
\hline & & B & A & K & G & N & D & C & & & & I & E & \\
\hline & & & B & A & M & G & L & D & C & & & & I & E \\
\hline X & & & & B & A & K & X & O & D & C & & & & X \\
\hline F & H & & & & B & A & P & J & Q & D & C & & & \\
\hline & F & H & & & & B & A & R & J & O & D & C & & \\
\hline & & F & H & & & & B & A & P & J & Q & D & C & \\
\hline & & & F & H & & & & B & A & R & J & O & D & C \\
\hline C & & & & F & H & & & & B & A & P & J & Q & D \\
\hline D & C & & & & F & H & & & & B & A & R & X & X \\
\hline X & D & C & & & & F & X & & & & B & A & X & X \\
\hline
\end{tabular}

Figure 3: Scheme of construction with $n=15$. 
Row 1. There are three ad hoc values plus the elements of the L, D, C, E, B and A diagonals. Namely:

$\mathcal{E}\left(\bar{R}_{1}\right)=(n-1,5 n, 9 n+2,-(8 n+2), n+2,-2 n,-(7 n+1), 6 n+1,-(5 n+1))$

and

$$
\mathcal{S}\left(\bar{R}_{1}\right)=(n-1,6 n-1,15 n+1,7 n-1,8 n+1,6 n+1,-n, 5 n+1,0) .
$$

Row 2. It is not hard to see that from the $K, \mathrm{G}, \mathrm{N}, \mathrm{D}, \mathrm{C}, \mathrm{I}, \mathrm{E}, \mathrm{B}$ and A diagonal cells we get:

$$
\begin{aligned}
\mathcal{E}\left(\bar{R}_{2}\right)=( & -(3 n+4),-(n-2), 4 n+1,9 n+3 \\
& -(8 n+3), 2 n-1,-(2 n-2),-(7 n+2), 6 n+2)
\end{aligned}
$$

and

$$
\mathcal{S}\left(\bar{R}_{2}\right)=(-(3 n+4),-(4 n+2),-1,9 n+2, n-1,3 n-2, n,-(6 n+2), 0) .
$$

Row 3. It is not hard to see that from the A, M, G, L, D, C, I, E and B diagonal cells we get:

$$
\begin{aligned}
\mathcal{E}\left(\bar{R}_{3}\right)= & (5 n+3,-(4 n+3),-(n-3), 5 n-1, \\
& 9 n+4,-(7 n+4), 2 n-3,-(2 n-4),-(7 n+3))
\end{aligned}
$$

and

$$
\mathcal{S}\left(\bar{R}_{3}\right)=(5 n+3, n, 3,5 n+2,14 n+6,7 n+2,9 n-1,7 n+3,0) .
$$

Row 4 to $\frac{n-1}{2}$. We have to distinguish two cases, depending on the parity of the row $r$. If $r$ is even, then write $r=4+2 i$ where $i \in\left[0, \frac{n-11}{4}\right]$. It is not hard to see that from the $\mathrm{B}, \mathrm{A}, \mathrm{K}, \mathrm{G}, \mathrm{N}, \mathrm{D}, \mathrm{C}, \mathrm{I}$ and $\mathrm{E}$ diagonal cells we get:

$$
\begin{aligned}
\mathcal{E}\left(\bar{R}_{4+2 i}\right)=( & (6 n+4+2 i), 5 n+4+2 i,-(3 n+5+i),-(n-4-2 i), \\
& 4 n-i, 8 n+5+2 i,-(7 n+5+2 i), 2 n-5-4 i,-(2 n-6-4 i))
\end{aligned}
$$

and

$$
\begin{aligned}
\mathcal{S}\left(\bar{R}_{4+2 i}\right)=( & -(6 n+4+2 i),-n,-(4 n+5+i), \\
& -(5 n+1-i),-(n+1), 7 n+4+2 i,-1,2 n-6-4 i, 0) .
\end{aligned}
$$

If $r$ is odd, then write $r=5+2 i$, where $i \in\left[0, \frac{n-11}{4}\right]$. It is not hard to see that from the B, A, M, G, L, D, C, I and E diagonal cells we get:

$$
\begin{aligned}
& \mathcal{E}\left(\bar{R}_{5+2 i}\right)=(-(6 n+5+2 i), 5 n+5+2 i,-(4 n+4+i),-(n-5-2 i), \\
&5 n-2-i, 8 n+6+2 i,-(7 n+6+2 i), 2 n-7-4 i,-(2 n-8-4 i))
\end{aligned}
$$

and

$$
\begin{aligned}
\mathcal{S}\left(\bar{R}_{5+2 i}\right)=( & -(6 n+5+2 i),-n,-(5 n+4+i) \\
& -(6 n-1-i),-(n+1), 7 n+5+2 i,-1,2 n-8-4 i, 0) .
\end{aligned}
$$


Row $\frac{n+1}{2}$. There are three ad hoc values plus the elements of the B, A, K, O, D and C diagonals. Namely:

$$
\begin{aligned}
\mathcal{E}\left(\bar{R}_{\frac{n+1}{2}}\right)= & -3 n,-\frac{13 n+1}{2}, \frac{11 n+1}{2} \\
& \left.-\frac{13 n+13}{4}, n, \frac{17 n+9}{4}, \frac{17 n+3}{2},-\frac{15 n+3}{2}, n+1\right)
\end{aligned}
$$

and

$$
\begin{aligned}
\mathcal{S}\left(\bar{R}_{\frac{n+1}{2}}\right)=( & -3 n,-\frac{19 n+1}{2},-4 n,-\frac{29 n+13}{4}, \\
& \left.-\frac{25 n+13}{4},-(2 n+1), \frac{13 n+1}{2},-(n+1), 0\right) .
\end{aligned}
$$

Row $\frac{n+3}{2}$ to $n-2$. We have to distinguish two cases, depending on the parity of the row $r$. If $r$ is odd, then write $r=\frac{n+3}{2}+2 i$ where $i \in\left[0, \frac{n-7}{4}\right]$. It is not hard to see that from the F, H, B, A, P, J, Q, D and C diagonal cells we get:

$$
\begin{gathered}
\mathcal{E}\left(\bar{R}_{\frac{n+3}{2}+2 i}\right)=\left(2 n+2+4 i,-(2 n+3+4 i),-\frac{13 n+3}{2}-2 i, \frac{11 n+3}{2}+2 i,\right. \\
\left.-\frac{15 n+7}{4}+i, \frac{n-3}{2}-2 i, \frac{13 n+17}{4}+i, \frac{17 n+5}{2}+2 i,-\frac{15 n+5}{2}-2 i\right)
\end{gathered}
$$

and

$$
\begin{aligned}
\mathcal{S}\left(\bar{R}_{\frac{n+3}{2}+2 i}\right)= & \left(2 n+2+4 i,-1,-\frac{13 n+5}{2}-2 i,-(n+1)\right. \\
& \left.\quad-\frac{19 n+11}{4}+i,-\frac{17 n+17}{4}-i,-n, \frac{15 n+5}{2}+2 i, 0\right) .
\end{aligned}
$$

If $r$ is even, then write $r=\frac{n+5}{2}+2 i$ where $i \in\left[0, \frac{n-11}{4}\right]$. It is not hard to see that from the $\mathrm{F}, \mathrm{H}, \mathrm{B}, \mathrm{A}, \mathrm{R}, \mathrm{J}, \mathrm{O}, \mathrm{D}$ and $\mathrm{C}$ diagonal cells we get:

$$
\begin{gathered}
\mathcal{E}\left(\bar{R}_{\frac{n+5}{2}+2 i}\right)=\left(2 n+4+4 i,-(2 n+5+4 i),-\frac{13 n+5}{2}-2 i, \frac{11 n+5}{2}+2 i,\right. \\
\left.-\frac{19 n-1}{4}+i, \frac{n-5}{2}-2 i, \frac{17 n+13}{4}+i, \frac{17 n+7}{2}+2 i,-\frac{15 n+7}{2}-2 i\right)
\end{gathered}
$$

and

$$
\begin{aligned}
\mathcal{S}\left(\bar{R}_{\frac{n+5}{2}+2 i}\right)=( & 2 n+4+4 i,-1,-\frac{13 n+7}{2}-2 i,-(n+1), \\
& \left.-\frac{23 n+3}{4}+i,-\frac{21 n+13}{4}-i,-n, \frac{15 n+7}{2}+2 i, 0\right) .
\end{aligned}
$$


Row $n-1$. There are two ad hoc values plus the elements of the D, C, F, H, B, A and R diagonals. Namely:

$$
\begin{aligned}
\mathcal{E}\left(\bar{R}_{n-1}\right)= & (9 n,-8 n, 3 n-3,-(3 n-2) \\
& \left.-(7 n-1), 6 n-1,-\frac{9 n+3}{2},-\frac{n-1}{2}, 5 n+2\right)
\end{aligned}
$$

and

$$
\mathcal{S}\left(\bar{R}_{n-1}\right)=\left(9 n, n, 4 n-3, n-1,-6 n,-1,-\frac{9 n+5}{2},-(5 n+2), 0\right) .
$$

Row $n$. There are four ad hoc values plus the elements of the D, C, F, B and A diagonals. Namely:

$\mathcal{E}\left(\bar{R}_{n}\right)=(3 n+3,9 n+1,-(8 n+1), 3 n-1,-(3 n+1),-7 n, 6 n,-(3 n+2), 1)$

and

$$
\mathcal{S}\left(\bar{R}_{n}\right)=(3 n+3,12 n+4,4 n+3,7 n+2,4 n+1,-(3 n-1), 3 n+1,-1,0) .
$$

Now we list the elements and the partial sums of the columns.

Column 1. There are three ad hoc values plus the elements of the $K, A, B, F, C$ and $D$ diagonals. Namely:

$\mathcal{E}\left(\bar{C}_{1}\right)=(n-1,-(3 n+4), 5 n+3,-(6 n+4),-3 n, 2 n+2,-(8 n-1), 9 n, 3 n+3)$

and

$$
\begin{aligned}
\mathcal{S}\left(\bar{C}_{1}\right)= & (n-1,-(2 n+5), 3 n-2,-(3 n+6), \\
& -(6 n+6),-(4 n+4),-(12 n+3),-(3 n+3), 0) .
\end{aligned}
$$

Column 2. It is not hard to see that from the $\mathrm{L}, \mathrm{G}, \mathrm{M}, \mathrm{A}, \mathrm{B}, \mathrm{H}, \mathrm{F}, \mathrm{C}$ and D diagonal cells we get:

$\mathcal{E}\left(\bar{C}_{2}\right)=(5 n,-(n-2),-(4 n+3), 5 n+4,-(6 n+5),-(2 n+3), 2 n+4,-8 n, 9 n+1)$

and

$\mathcal{S}\left(\bar{C}_{2}\right)=(5 n, 4 n+2,-1,5 n+3,-(n+2),-(3 n+5),-(n+1),-(9 n+1), 0)$.

Column 3. It is not hard to see that from the $\mathrm{D}, \mathrm{N}, \mathrm{G}, \mathrm{K}, \mathrm{A}, \mathrm{B}, \mathrm{H}, \mathrm{F}$ and $\mathrm{C}$ diagonal cells we get:

$$
\begin{aligned}
\mathcal{E}\left(\bar{C}_{3}\right)= & (9 n+2,4 n+1,-(n-3),-(3 n+5), \\
& 5 n+5,-(6 n+6),-(2 n+5), 2 n+6,-(8 n+1))
\end{aligned}
$$

and

$$
\mathcal{S}\left(\bar{C}_{3}\right)=(9 n+2,13 n+3,12 n+6,9 n+1,14 n+6,8 n, 6 n-5,8 n+1,0) .
$$


Column 4. It is not hard to see that from the C, D, L, G, M, A, B, H and F diagonal cells we get:

$$
\begin{aligned}
\mathcal{E}\left(\bar{C}_{4}\right)=( & -(8 n+2), 9 n+3,5 n-1,-(n-4), \\
& -(4 n+4), 5 n+6,-(6 n+7),-(2 n+7), 2 n+8)
\end{aligned}
$$

and

$$
\mathcal{S}\left(\bar{C}_{4}\right)=(-(8 n+2), n+1,6 n, 5 n+4, n, 6 n+6,-1,-(2 n+8), 0) .
$$

Column 5. It is not hard to see that from the C, D, N, G, K, A, B, H and F diagonal cells we get:

$$
\begin{aligned}
\mathcal{E}\left(\bar{C}_{5}\right)=( & -(8 n+3), 9 n+4,4 n,-(n-5), \\
& -(3 n+6), 5 n+7,-(6 n+8),-(2 n+9), 2 n+10)
\end{aligned}
$$

and

$$
\mathcal{S}\left(\bar{C}_{5}\right)=(-(8 n+3), n+1,5 n+1,4 n+6, n, 6 n+7,-1,-(2 n+10), 0) .
$$

Column 6 to $\frac{n-1}{2}$. We have to distinguish two cases, depending on the parity of the column $c$. If $c$ is even, then write $c=6+2 i$ where $i \in\left[0, \frac{n-15}{4}\right]$. It is not hard to see that from the $\mathrm{C}, \mathrm{D}, \mathrm{L}, \mathrm{G}, \mathrm{M}, \mathrm{A}, \mathrm{B}, \mathrm{H}$ and $\mathrm{F}$ diagonal cells we get:

$$
\begin{aligned}
& \mathcal{E}\left(\bar{C}_{6+2 i}\right)=(-(7 n+4+2 i), 8 n+5+2 i, 5 n-2-i,-(n-6-2 i), \\
& \quad-(4 n+5+i), 5 n+8+2 i,-(6 n+9+2 i),-(2 n+11+4 i), 2 n+12+4 i)
\end{aligned}
$$

and

$$
\begin{aligned}
\mathcal{S}\left(\bar{C}_{6+2 i}\right)= & (-(7 n+4+2 i), n+1,6 n-1-i, \\
& 5 n+5+i, n, 6 n+8+2 i,-1,-(2 n+12+4 i), 0) .
\end{aligned}
$$

If $c$ is odd, then write $c=7+2 i$ where $i \in\left[0, \frac{n-15}{4}\right]$. It is not hard to see that from the $\mathrm{C}, \mathrm{D}, \mathrm{N}, \mathrm{G}, \mathrm{K}, \mathrm{A}, \mathrm{B}, \mathrm{H}$ and $\mathrm{F}$ diagonal cells we get:

$$
\begin{aligned}
& \mathcal{E}\left(\bar{C}_{7+2 i}\right)=(-(7 n+5+2 i), 8 n+6+2 i, 4 n-1-i,-(n-7-2 i), \\
& \quad-(3 n+7+i), 5 n+9+2 i,-(6 n+10+2 i),-(2 n+13+4 i), 2 n+14+4 i)
\end{aligned}
$$

and

$$
\begin{aligned}
\mathcal{S}\left(\bar{C}_{7+2 i}\right)= & (-(7 n+5+2 i), n+1,5 n-i, \\
& 4 n+7+i, n, 6 n+9+2 i,-1,-(2 n+14+4 i), 0) .
\end{aligned}
$$

Column $\frac{n+1}{2}$. The are three ad hoc values plus the elements of the C, D, L, P, A and B diagonals. Namely:

$$
\begin{aligned}
\mathcal{E}\left(\bar{C}_{\frac{n+1}{2}}\right)= & \left(n+2,-\frac{15 n-3}{2}, \frac{17 n-1}{2}, \frac{19 n+3}{4},\right. \\
& \left.n,-\frac{15 n+7}{4}, \frac{11 n+5}{2},-\frac{13 n+7}{4},-(3 n+1)\right)
\end{aligned}
$$


and

$$
\begin{aligned}
\mathcal{S}\left(\bar{C}_{\frac{n+1}{2}}\right)= & \left(n+2,-\frac{13 n-7}{2}, 2 n+3,\right. \\
& \left.\frac{27 n+15}{4}, \frac{31 n+1}{4}, 4 n+2, \frac{19 n+9}{2}, 3 n+1,0\right) .
\end{aligned}
$$

Column $\frac{n+3}{2}$ to $n-2$. We have to distinguish two cases, depending on the parity of the column $c$. If $c$ is odd, then write $c=\frac{n+3}{2}+2 i$ where $i \in\left[0, \frac{n-7}{4}\right]$. It is not hard to see that from the E, I, C, D, O, J, R, A and B diagonal cells we get:

$$
\begin{gathered}
\mathcal{E}\left(\bar{C}_{\frac{n+3}{2}+2 i}\right)=\left(-(2 n-4 i), 2 n-1-4 i,-\frac{15 n-1}{2}-2 i, \frac{17 n+1}{2}+2 i,\right. \\
\left.\quad \frac{17 n+9}{4}+i, \frac{n-3}{2}-2 i,-\frac{19 n-1}{4}+i, \frac{11 n+7}{2}+2 i,-\frac{13 n+9}{2}-2 i,\right)
\end{gathered}
$$

and

$$
\begin{aligned}
\mathcal{S}\left(\bar{C}_{\frac{n+3}{2}+2 i}\right)=( & -(2 n-4 i),-1,-\frac{15 n+1}{2}-2 i \\
& \left.n, \frac{21 n+9}{4}+i, \frac{23 n+3}{4}-i, n+1, \frac{13 n+9}{2}+2 i, 0\right) .
\end{aligned}
$$

If $c$ is even, then write $c=\frac{n+5}{2}+2 i$ where $i \in\left[0, \frac{n-11}{4}\right]$. It is not hard to see that from the E, I, C, D, Q, J, P, A and B diagonal cells we get:

$$
\begin{gathered}
\mathcal{E}\left(\bar{C}_{\frac{n+5}{2}+2 i}\right)=\left(-(2 n-2-4 i), 2 n-3-4 i,-\frac{15 n+1}{2}-2 i, \frac{17 n+3}{2}+2 i,\right. \\
\left.\frac{13 n+17}{4}+i, \frac{n-5}{2}-2 i,-\frac{15 n+3}{4}+i, \frac{11 n+9}{2}+2 i,-\frac{13 n+11}{2}-2 i\right)
\end{gathered}
$$

and

$$
\begin{aligned}
\mathcal{S}\left(\bar{C}_{\frac{n+5}{2}+2 i}\right)= & \left(-(2 n-2-4 i),-1,-\frac{15 n+3}{2}, n, \frac{17 n+17}{4}+i\right. \\
& \left.\frac{19 n+7}{4}-i, n+1, \frac{13 n+11}{2}+2 i, 0\right)
\end{aligned}
$$

Column $n-1$. There are two ad hoc values plus the elements of the A, B, E, I, C, D and $Q$ diagonals. Namely:

$$
\begin{aligned}
\mathcal{E}\left(\bar{C}_{n-1}\right)= & (6 n+1,-(7 n+2),-(n+5), n+4 \\
& \left.-(8 n-3), 9 n-2, \frac{7 n+5}{2},-\frac{n-1}{2},-(3 n+2)\right)
\end{aligned}
$$

and

$$
\begin{aligned}
\mathcal{S}\left(\bar{C}_{n-1}\right)= & (6 n+1,-(n+1),-(2 n+6), \\
& \left.\quad-(n+2),-(9 n-1),-1, \frac{7 n+3}{2}, 3 n+2,0\right) .
\end{aligned}
$$


Column $n$. There are four ad hoc values plus the elements of the A, B, E, C and D diagonals. Namely:

$$
\mathcal{E}\left(\bar{C}_{n}\right)=(-(5 n+1), 6 n+2,-(7 n+3),-(n+3), n+1,-(8 n-2), 9 n-1,5 n+2,1)
$$

and

$$
\begin{aligned}
S\left(\bar{C}_{n}\right)=( & -(5 n+1), n+1,-(6 n+2),-(7 n+5), \\
& -(6 n+4),-(14 n+2),-(5 n+3),-1,0) .
\end{aligned}
$$

Finally, we consider the support of $A$ :

$$
\begin{aligned}
\operatorname{supp}(A)= & \{1\} \cup\left[2, \frac{n-3}{2}\right]_{(\mathrm{J})} \cup\left\{\frac{n-1}{2}\right\} \cup\left[\frac{n+1}{2}, n-2\right]_{(\mathrm{G})} \\
& \cup\{n-1, n, n+1, n+2\} \cup[n+3,2 n]_{(\mathrm{E} \cup \mathrm{I})} \cup[2 n+2,3 n-1]_{(\mathrm{F} \cup \mathrm{H})} \\
& \cup\{3 n, 3 n+1,3 n+2,3 n+3\} \cup\left[3 n+4, \frac{13 n+13}{4}\right]_{(\mathrm{K})} \\
& \cup\left[\frac{13 n+17}{4}, \frac{7 n+5}{2}\right]_{(\mathrm{Q})} \cup\left[\frac{7 n+7}{2}, \frac{15 n+7}{4}\right]_{(\mathrm{P})} \cup\left[\frac{15 n+11}{4}, 4 n+1\right]_{(\mathrm{N})} \\
& \cup\left[4 n+3, \frac{17 n+5}{4}\right]_{(\mathrm{M})} \cup\left[\frac{17 n+9}{4}, \frac{9 n+1}{2}\right]_{(\mathrm{O})} \cup\left[\frac{9 n+3}{2}, \frac{19 n-1}{4}\right]_{(\mathrm{R})} \\
& \cup\left[\frac{19 n+3}{4}, 5 n\right]_{(\mathrm{L})} \cup\{5 n+1,5 n+2\} \cup[5 n+3,6 n+2]_{(\mathrm{A})} \\
& \cup[6 n+4,7 n+3]_{(\mathrm{B})} \cup[7 n+4,8 n+3]_{(\mathrm{C})} \cup[8 n+5,9 n+4]_{(\mathrm{D})} \\
= & {[1,9 n+4] \backslash\{2 n+1,4 n+2,6 n+3,8 n+4\} . }
\end{aligned}
$$

This concludes the proof.

Example 4.9. Following the proof of Proposition 4.8 we obtain the integer globally simple $\mathrm{H}_{9}(15 ; 9)$ given in Figure 4.

Lemma 4.10. For any $n \equiv 7(\bmod 14)$ such that $n \geq 21$, write $r=\frac{n-7}{2}$. Let $A_{n}$ be a 9 diagonal array whose filled diagonals are $D_{1}, D_{2}, \ldots, D_{7}, D_{r+7}$ and $D_{r+8}$. Then $(\mathcal{R}, \mathcal{C})$, where $\mathcal{R}=(1,1, \ldots, 1)$ and $\mathcal{C}=(\underbrace{-1, \ldots,-1}_{8}, 1,1, \ldots, 1)$, is a solution of $P\left(A_{n}\right)$.

Proof. For any $i \in[1,7] \cup\{r+7, r+8\}$ set $D_{i}=\left(d_{i, 1}, d_{i, 2}, d_{i, 3}, \ldots, d_{i, n}\right)$, where $d_{i, 1}$ is the position $[i, 1]$ of $A_{n}$. Also, we set

$$
\begin{aligned}
& \mathbf{A}_{i}=d_{i, 8}, d_{i, 9}, d_{i, 10}, \ldots, d_{i, n} ; \\
& \mathbf{B}_{i}=d_{1, i}, d_{1, i+r}, d_{1, i+2 r}, \ldots, d_{1, i+\frac{2 r}{7} r} ; \\
& \mathbf{C}_{i}=d_{r+7, i}, d_{r+7, i+r}, d_{r+7, i+2 r}, \ldots, d_{r+7, i+\frac{2 r}{7} r} ; \\
& \mathbf{D}_{1}=d_{1,1}, d_{1,1+r}, d_{1,1+2 r}, \ldots, d_{1,1+\left(\frac{2 r}{7}-2\right) r} ; \\
& \mathbf{D}_{2}=d_{1,8}, d_{1,8+r} ; \\
& \mathbf{E}_{1}=d_{r+7,1}, d_{r+7,1+r}, d_{r+7,1+2 r}, \ldots, d_{r+7,1+\left(\frac{2 r}{7}-2\right) r} \\
& \mathbf{E}_{2}=d_{r+7,8}, d_{r+7,8+r} .
\end{aligned}
$$




\begin{tabular}{|c|c|c|c|c|c|c|c|c|c|c|c|c|c|}
\hline$\stackrel{0}{\stackrel{0}{1}}$ & ฮิ & $\begin{array}{l}\infty \\
\stackrel{\infty}{=} \\
\rightarrow \\
\end{array}$ & & & & & $\stackrel{0}{-1}$ & & & & & $\vec{O}$ & N \\
\hline$s$ & $\begin{array}{l}\stackrel{\sim}{0} \\
\stackrel{-}{1} \\
\end{array}$ & & & & $\stackrel{\curvearrowright}{\curvearrowright}$ & $\stackrel{2}{-}$ & & & & $\underset{1}{=} \underset{1}{=}$ & & & 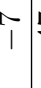 \\
\hline & & & & $\underset{\sim}{\mathbb{N}}$ & $\overrightarrow{\mathrm{N}}$ & & & & $\begin{array}{l}0 \\
\underset{-}{-} \\
1\end{array}$ & $\underset{\overbrace{}}{\stackrel{1}{\rightarrow}}$ & 8 & $\sim$ & $\begin{array}{l}\stackrel{0}{0} \\
1\end{array}$ \\
\hline & & & $\underset{\sim}{\sim}$ & $\stackrel{\Re}{\ominus}$ & & & & 足 & $\vec{m}$ & $\Delta 0^{+1}$ & & $\begin{array}{c}0 \\
20 \\
10\end{array}$ & $\infty$ \\
\hline & & 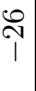 & $\stackrel{\stackrel{2}{N}}{\sim}$ & & & & $\underset{\vec{I}}{\vec{T}}$ & $\stackrel{\leftrightarrow}{\stackrel{\oplus}{-}}$ & 6 & $F$ & & $\begin{array}{l}\infty \\
\infty \\
\infty\end{array}$ & $\begin{array}{l}\overrightarrow{1} \\
\stackrel{1}{1} \\
\stackrel{1}{1}\end{array}$ \\
\hline & $\begin{array}{c}\stackrel{\infty}{\sim} \\
\stackrel{\imath}{\imath}\end{array}$ & $\stackrel{\sim}{N}$ & & & & $\stackrel{2}{\rightarrow}$ & $\stackrel{尺}{\stackrel{I}{-}}$ & జึ & 10 & $\overbrace{10}^{10}$ & & $\stackrel{\substack{0 \\
1}}{1}$ & \\
\hline 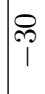 & $\stackrel{\vec{i}}{ }$ & & & & $\underset{\sim}{\stackrel{\sim}{\nearrow}}$ & 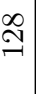 & $\bullet$ & 0 & $\stackrel{\Gamma}{\Gamma}$ & 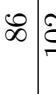 & & & \\
\hline$\stackrel{\sim}{-1}$ & & & & $\vec{\exists}$ & $\stackrel{N}{\sim}$ & $\stackrel{N}{i}$ & $\stackrel{10}{-1}$ & 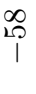 & $\infty 2$ & $\begin{array}{l}\vec{\sigma} \\
\stackrel{1}{1}\end{array}$ & & & \\
\hline & & & $\underset{\mathrm{O}}{\stackrel{0}{二}}$ & 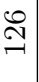 & $\therefore 8$ & $\begin{array}{c}\infty \\
1\end{array}$ & î & $\vec{\infty}^{\prime}$ & $\begin{array}{l}8 \\
\stackrel{-}{1}\end{array}$ & & & & $\stackrel{\Re}{+}$ \\
\hline & & $\begin{array}{c}8 \\
\stackrel{0}{0} \\
1\end{array}$ & $\stackrel{\stackrel{s}{N}}{\sim}$ & $\Re$ & $i$ & $\begin{array}{l}10 \\
0 \\
1\end{array}$ & $\infty$ & \& & & & & $\vec{F}$ & $\mathscr{F}$ \\
\hline & $\begin{array}{l}\stackrel{\Re}{\underset{T}{T}} \\
\end{array}$ & $\stackrel{\Leftrightarrow}{\stackrel{\oplus}{-}}$ & 8 & $\stackrel{0}{\rightarrow}$ & $\overrightarrow{20}$ & $\infty$ & $\infty$ & & & ๙ & & 아 & \\
\hline 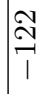 & $\begin{array}{l}\infty \\
\infty \\
-\end{array}$ & 点 & $\underset{7}{1}$ & $\begin{array}{c}\overrightarrow{0} \\
1\end{array}$ & $\infty$ & $\hat{\sigma}$ & & & & 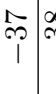 & 冓 & & \\
\hline$\stackrel{\sim}{\stackrel{N}{=}}$ & $\overline{6}$ & $\stackrel{\mathcal{F}}{\mathcal{T}}$ & $\stackrel{8}{20}$ & 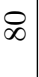 & $\stackrel{8}{\circ}$ & & & & $\begin{array}{c}20 \\
i \\
i\end{array}$ & $\stackrel{\circ}{\circ}$ & & & \\
\hline$\stackrel{20}{12}$ & $\stackrel{m}{\rightarrow}$ & $\begin{array}{l}3 \\
0 \\
1\end{array}$ & 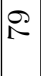 & $\stackrel{2}{2}$ & & & & \begin{tabular}{c}
$\infty$ \\
\hdashline \\
$i$ \\
$i$
\end{tabular} & के & & & & $\stackrel{\curvearrowright}{\mathcal{N}}$ \\
\hline$\exists$ & 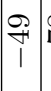 & $\stackrel{\infty}{1}$ & $\overrightarrow{0}$ & & & & $\stackrel{\stackrel{2}{*}}{1}$ & ळै & & & & 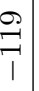 & 号 \\
\hline
\end{tabular}

Figure 4: An integer globally simple $\mathrm{H}_{9}(15 ; 9)$. 
To aid in the proof, at the webpage

http://anita-pasotti.unibs.it/Publications.html,

we give a schematic picture of where each of these sequences fills cells. By a direct check, one can verify that

$$
\begin{aligned}
L_{\mathcal{R}, \mathcal{C}}\left(d_{6,8}\right)= & \left(\mathbf{A}_{6}, d_{4,1}, d_{2,2}, d_{r+8,3}, d_{7,4}, d_{5,5}, d_{3,6}, \mathbf{B}_{7}, \mathbf{C}_{7}, d_{6,7}\right. \\
& \mathbf{A}_{4}, d_{2,1}, d_{r+8,2}, d_{7,3}, d_{5,4}, d_{3,5}, \mathbf{B}_{6}, \mathbf{C}_{6}, d_{6,6}, d_{4,7} \\
& \mathbf{A}_{2}, d_{r+8,1}, d_{7,2}, d_{5,3}, d_{3,4}, \mathbf{B}_{5}, \mathbf{C}_{5}, d_{6,5}, d_{4,6}, d_{2,7} \\
& \mathbf{A}_{r+8}, d_{7,1}, d_{5,2}, d_{3,3}, \mathbf{B}_{4}, \mathbf{C}_{4}, d_{6,4}, d_{4,5}, d_{2,6}, d_{r+8,7} \\
& \mathbf{A}_{7}, d_{5,1}, d_{3,2}, \mathbf{B}_{3}, \mathbf{C}_{3}, d_{6,3}, d_{4,4}, d_{2,5}, d_{r+8,6}, d_{7,7} \\
& \mathbf{A}_{5}, d_{3,1}, \mathbf{B}_{2}, \mathbf{C}_{2}, d_{6,2}, d_{4,3}, d_{2,4}, d_{r+8,5}, d_{7,6}, d_{5,7}, \\
& \left.\mathbf{A}_{3}, \mathbf{D}_{1}, \mathbf{E}_{2}, d_{6,1}, d_{4,2}, d_{2,3}, d_{r+8,4}, d_{7,5}, d_{5,6}, d_{3,7}, \mathbf{D}_{2}, \mathbf{E}_{1}\right) .
\end{aligned}
$$

Hence, it is easy to see that $L_{\mathcal{R}, \mathcal{C}}\left(d_{6,8}\right)$ covers all the filled cells of $A_{n}$.

Now we are ready to prove Theorem 1.3.

Proof of Theorem 1.3. The result follows from Theorem 3.4, once we have proved the existence of a relative Heffter array with compatible simple orderings $\omega_{r}$ and $\omega_{c}$.

(1): For any $n$ odd, a $\mathrm{H}_{n}(n ; 3)$ and a $\mathrm{H}_{2 n}(n ; 3)$ are constructed in Propositions 4.1 and 4.3 , respectively. Clearly these are globally simple Heffter arrays. Since they are cyclically 3-diagonal their compatibility follows from [13, Proposition 3.4].

(2): Let $n \equiv 3(\bmod 4) . \mathrm{A} \mathrm{H}_{3}(n ; 3)$ and a $\mathrm{H}_{5}(n ; 5)$ are constructed in [15, Propositions 5.1 and 5.5], respectively. As before these are globally simple Heffter arrays and since they are cyclically 3 -diagonal and 5-diagonal, respectively, their compatibility follows from [13, Proposition 3.4]. A globally simple $\mathrm{H}_{7}(n ; 7)$ is given in Proposition 4.6. Since this is cyclically 7-diagonal its compatibility follows from [13, Propositions 3.4 and 3.6]. Finally, a globally simple $\mathrm{H}_{9}(n ; 9)$ is given in Proposition 4.8. Since this is 9-diagonal with width $\frac{n-9}{2}$, if $\operatorname{gcd}\left(n, \frac{n-7}{2}\right)=\operatorname{gcd}(n, 7)=1$ its compatibility follows from [12, Proposition 4.19]. If $\operatorname{gcd}(n, 7) \neq 1$ the result follows from Lemma 4.10.

\section{Archdeacon arrays}

In this section we introduce a further generalization of the concept of Heffter array. In particular we will consider p.f. arrays where the number of filled cells in each row and in each column is not fixed.

Definition 5.1. An Archdeacon array $A$ over an abelian group $(G,+)$ is an $m \times n$ p.f. array with elements in $G$, such that:

(a) $\mathcal{E}(A)$ is a set;

(b) for every $g \in G, g \in \mathcal{E}(A)$ implies $-g \notin \mathcal{E}(A)$;

(c) the elements in every row and column sum to 0 .

An example of this kind of arrays will be given in Figure 5. We note that, in the special case $G=\mathbb{Z}_{v}, \pm \mathcal{E}(A)=\mathbb{Z}_{v} \backslash J$ where $J$ is a subgroup of $\mathbb{Z}_{v}$ and all the rows (resp. columns) have the same number of filled cells, we meet again the definition of a relative 
Heffter array. The purpose of this section is to show how Archdeacon arrays can be used in order to obtain biembeddings and orthogonal cycle decompositions. First of all we need a generalization of [7, Proposition 2.6], stated by Buratti in [6, Theorem 3.3]. All the well known concepts about the differences method can be found in $[6,15]$.

Theorem 5.2. Let $G$ be an additive group and $\mathcal{B}$ be a set of cycles with vertices in $G$. If the list of differences of $\mathcal{B}$ is a set, say $\Lambda$, then $\mathcal{B}$ is a set of base cycles of a $G$-regular cycle decomposition of Cay $[G: \Lambda]$.

Generalizing Proposition 2.1, an Archdeacon array can be used to obtain regular cycle decompositions of Cayley graphs as follows.

Proposition 5.3. Let $A$ be an $m \times n$ Archdeacon array on an abelian group $G$ with simple orderings $\omega_{r}=\omega_{\bar{R}_{1}} \circ \cdots \circ \omega_{\bar{R}_{m}}$ for the rows and $\omega_{c}=\omega_{\bar{C}_{1}} \circ \cdots \circ \omega_{\bar{C}_{n}}$ for the columns. Then:

(1) $\mathcal{B}_{\omega_{r}}=\left\{\mathcal{S}\left(\omega_{\bar{R}_{i}}\right) \mid i \in[1, m]\right\}$ is a set of base cycles of a $G$-regular cycle decomposition $\mathcal{D}_{\omega_{r}}$ of Cay $[G: \pm \mathcal{E}(A)]$;

(2) $\mathcal{B}_{\omega_{c}}=\left\{\mathcal{S}\left(\omega_{\bar{C}_{j}}\right) \mid j \in[1, n]\right\}$ is a set of base cycles of a $G$-regular cycle decomposition $\mathcal{D}_{\omega_{c}}$ of Cay $[G: \pm \mathcal{E}(A)]$;

(3) the cycle decompositions $\mathcal{D}_{\omega_{r}}$ and $\mathcal{D}_{\omega_{c}}$ are orthogonal.

Proof. (1): Since the ordering $\omega_{r}$ is simple the elements of $\mathcal{B}_{\omega_{r}}$ are cycles of lengths $\left|\mathcal{E}\left(\bar{R}_{1}\right)\right|, \ldots,\left|\mathcal{E}\left(\bar{R}_{m}\right)\right|$ and by definition of partial sums the list of differences of $\mathcal{S}\left(\omega_{\bar{R}_{i}}\right)$ is $\pm \mathcal{E}\left(\bar{R}_{i}\right)$, for any $i \in[1, m]$. Hence, the list of differences of $\mathcal{B}_{\omega_{r}}$ is $\pm \mathcal{E}(A)$ and so the thesis follows from Theorem 5.2. Obviously, (2) can be proved in the same way. Note that, in general, the cycles of $\mathcal{B}_{\omega_{r}}$ and those of $\mathcal{B}_{\omega_{c}}$ have different lengths. (3) follows from the requirement that the elements of $\pm \mathcal{E}(A)$ are pairwise distinct.

Moreover the pair of cycles decompositions obtained from an Archdeacon array can be biembedded under the same hypothesis of Theorem 3.4. In fact, within the same proof, we have that:

Theorem 5.4. Let $A$ be an Archdeacon array on an abelian group $G$ that is simple with respect to two compatible orderings $\omega_{r}$ and $\omega_{c}$. Then there exists a biembedding of the $G$-regular cycle decompositions $\mathcal{D}_{\omega_{r}^{-1}}$ and $\mathcal{D}_{\omega_{c}}$ of $\mathrm{Cay}[G: \pm \mathcal{E}(A)]$ into an orientable surface.

We observe that if an Archdeacon array has no empty rows/columns, then a necessary condition for the existence of compatible orderings is $|\operatorname{skel}(A)| \equiv m+n-1(\bmod 2)$. This can be proved with the same proof of [11, Theorem 1.1] and of [12, Theorem 2.7].

Finally, as an easy consequence of Theorem 5.4, we obtain the relationship between the Crazy Knight's Tour Problem and globally simple Archdeacon arrays.

Corollary 5.5. Let $A$ be a globally simple Archdeacon array on an abelian group $G$ such that $P(A)$ admits a solution $(\mathcal{R}, \mathcal{C})$. Then there exists a biembedding of the $G$-regular cycle decompositions $\mathcal{D}_{\omega_{r}^{-1}}$ and $\mathcal{D}_{\omega_{c}}$ of $\mathrm{Cay}[G: \pm \mathcal{E}(A)]$ into an orientable surface. 
Given two $m \times n$ p.f. arrays $A$ and $B$ defined on abelian groups $G_{1}$ and $G_{2}$, respectively, we define their direct sum $A \oplus B$ as the $m \times n$ p.f. array $E$ whose skeleton is $\operatorname{skel}(A) \cup$ $\operatorname{skel}(B)$ and whose entries in $G_{1} \oplus G_{2}$ are so defined:

$$
E[i, j]= \begin{cases}(A[i, j], B[i, j]) & \text { if }(i, j) \in \operatorname{skel}(A) \cap \operatorname{skel}(B), \\ \left(A[i, j], 0_{G_{2}}\right) & \text { if }(i, j) \in \operatorname{skel}(A) \backslash \operatorname{skel}(B), \\ \left(0_{G_{1}}, B[i, j]\right) & \text { if }(i, j) \in \operatorname{skel}(B) \backslash \operatorname{skel}(A) .\end{cases}
$$

In the following we will denote by $\bar{R}_{i}(A)$ and $\bar{C}_{j}(A)$ the $i$-th row and the $j$-th column of $A$, respectively.

Lemma 5.6. Let $A$ and $B$ be $m \times n$ globally simple p.f. arrays over abelian groups $G_{1}$ and $G_{2}$, respectively, such that:

(1) for any $i \in[1, m]$ for which the $i$-th rows of $A$ and $B$ are both nonempty, we have $\operatorname{skel}\left(\bar{R}_{i}(A)\right) \cap \operatorname{skel}\left(\bar{R}_{i}(B)\right) \neq \emptyset$;

(2) for any $j \in[1, n]$ for which the $j$-th columns of $A$ and $B$ are both nonempty, we have $\operatorname{skel}\left(\bar{C}_{j}(A)\right) \cap \operatorname{skel}\left(\bar{C}_{j}(B)\right) \neq \emptyset$;

(3) the elements in every nonempty row/column of both $A$ and $B$ sum to zero.

Then $A \oplus B$ is a globally simple p.f. array, whose nonempty rows and columns sum to zero.

Proof. Since the elements in every nonempty row and column of both $A$ and $B$ sum to zero, the same holds for $A \oplus B$.

Let us suppose, by contradiction, that there exists a row (resp. a column) $\bar{R}_{i}$ of $A \oplus B$ that is not simple with respect to the natural ordering. Then there would be a subsequence $L$ of consecutive elements of $\bar{R}_{i}$ that sum to zero. Denoted by $L_{1}$ the subsequence of the first coordinates of $L$ (ignoring the zeros) and by $L_{2}$ the one of the second coordinates, we have that both $L_{1}$ and $L_{2}$ sums to zero. Since both $\bar{R}_{i}(A)$ and $\bar{R}_{i}(B)$ are simple with respect to the natural ordering, it follows that either $L_{1}=\emptyset$ (we are ignoring zeros) or $L_{1}=\mathcal{E}(A)$. Similarly, for $\bar{R}_{i}(B)$. If $L_{1}=\emptyset$, then $L_{2}=\mathcal{E}\left(\bar{R}_{i}(B)\right)$ and hence $L$ is $\mathcal{E}\left(\bar{R}_{i}\right)$. Similarly, if $L_{2}=\emptyset$. Finally, if $L_{1}$ and $L_{2}$ are both nonempty, the only possibility is that $L=\mathcal{E}\left(\bar{R}_{i}\right)$ since $\operatorname{skel}\left(\bar{R}_{i}(A)\right) \cap \operatorname{skel}\left(\bar{R}_{i}(B)\right) \neq \emptyset$.

Proposition 5.7. Let $A$ be an Archdeacon array over an abelian group $G_{1}$ and let $B$ be a p.f. array of the same size defined over an abelian group $G_{2}$. Suppose that the hypotheses of Lemma 5.6 are satisfied, that $\mathcal{E}(A \oplus B)$ is a set and that if $\left(0_{G_{1}}, x\right) \in \mathcal{E}(A \oplus B)$, then $\left(0_{G_{1}},-x\right) \notin \mathcal{E}(A \oplus B)$. Then $A \oplus B$ is a globally simple Archdeacon array over $G_{1} \oplus G_{2}$.

Proof. By Lemma 5.6, $E=A \oplus B$ is a globally simple p.f. array whose rows and columns sum to zero. We now show that condition (b) of Definition 5.1 holds. Suppose that $g=$ $\left(g_{1}, g_{2}\right) \in G_{1} \oplus G_{2}$ belongs to $\mathcal{E}(E)$. Then, either $g_{1} \in \mathcal{E}(A)$ or $g_{1}=0_{G_{1}}$. In the first case, $-g_{1} \notin \mathcal{E}(A)$ and so $-g=\left(-g_{1},-g_{2}\right) \notin \mathcal{E}(E)$. If $g_{1}=0_{G_{1}}$, then $\left(0_{G_{1}},-g_{2}\right) \notin \mathcal{E}(E)$ by hypothesis, proving the statement.

Now we consider the $m \times n$ p.f. array $B_{m, n, d}\left(i_{1}, i_{2} ; j_{1}, j_{2}\right)$ over $\mathbb{Z}_{d}$ which has only four nonempty cells: those in positions $\left(i_{1}, j_{1}\right),\left(i_{2}, j_{2}\right)$ that we fill with +1 and those in positions $\left(i_{2}, j_{1}\right),\left(i_{1}, j_{2}\right)$ that we fill with -1 . The following result is a consequence of Proposition 5.7. 
Corollary 5.8. Let $k<n$ and let us suppose there exists a globally simple cyclically $k$ diagonal $\mathrm{H}_{t}(n ; k)$, say $A$, whose filled diagonals are $D_{1}, \ldots, D_{k}$. Then considering the array $B=B_{n, n, d}(1,2 ; 1,2)$, where $d>2$, we have that $E=A \oplus B$ is a globally simple Archdeacon array over the group $\mathbb{Z}_{2 n k+t} \oplus \mathbb{Z}_{d}$.

We know that there exists a (globally simple) cyclically 3 -diagonal $\mathrm{H}_{t}(n ; 3)$ in each of the following cases:

(1) $t \in\{1,2\}$ and $n \equiv 0,1(\bmod 4)$, see [4, Theorems 3.4 and 3.9];

(2) $t=3$ and $n \equiv 0,3(\bmod 4)$, see [15, Propositions 5.1 and 5.3];

(3) $t=n$ and $n$ is odd, see Proposition 4.1;

(4) $t=2 n$ and $n$ is odd, see Proposition 4.3.

Therefore in these cases, we can apply Corollary 5.8: for any $d \geq 3$ there exists a globally simple Archdeacon array $E$ of size $n \geq 4$ defined over $\mathbb{Z}_{6 n+t} \oplus \mathbb{Z}_{d}$ whose skeleton is $D_{1} \cup D_{2} \cup D_{3} \cup\{(1,2)\}$.

Moreover, because of [12, Proposition 5.9], there exists a solution of $P(E)$ whenever $n$ is also even. In those cases we have a biembedding of Cay $\left[\mathbb{Z}_{6 n+t} \oplus \mathbb{Z}_{d}: \pm \mathcal{E}(E)\right]$ in an orientable surface whose faces classes contain triangles and exactly one quadrangle.

As example of such construction, in Figure 5 we give a globally simple Archdeacon array over $\mathbb{Z}_{51} \oplus \mathbb{Z}_{d}$, where $d \geq 3$.

\begin{tabular}{|c|c|c|c|c|c|c|c|}
\hline$(-9,1)$ & $(0,-1)$ & & & & & $(16,0)$ & $(-7,0)$ \\
\hline$(-3,-1)$ & $(-22,1)$ & & & & & & $(25,0)$ \\
\hline$(12,0)$ & $(1,0)$ & $(-13,0)$ & & & & & \\
\hline & $(21,0)$ & $(2,0)$ & $(-23,0)$ & & & & \\
\hline & & $(11,0)$ & $(8,0)$ & $(-19,0)$ & & & \\
\hline & & & $(15,0)$ & $(5,0)$ & $(-20,0)$ & & \\
\hline & & & & $(14,0)$ & $(-4,0)$ & $(-10,0)$ & \\
\hline & & & & & $(24,0)$ & $(-6,0)$ & $(-18,0)$ \\
\hline
\end{tabular}

Figure 5: An Archdeacon array over $\mathbb{Z}_{51} \oplus \mathbb{Z}_{d}$.

We recall that the existence of a (globally simple) cyclically 4-diagonal $\mathrm{H}_{t}(n ; 4)$ for any $n$ and $t \in\{1,2,4\}$ has been proved in [17, Theorem 2.2] and [15, Proposition 4.9]. Therefore, for any $d \geq 3$, because of Corollary 5.8 there exists a globally simple Archdeacon array $E$ of size $n \geq 4$ over $\mathbb{Z}_{8 n+t} \oplus \mathbb{Z}_{d}$ whose skeleton is $D_{1} \cup D_{2} \cup D_{3} \cup D_{4} \cup\{(1,2)\}$.

Moreover, because of [12, Proposition 5.13], there exists a solution of $P(E)$ whenever $n \not \equiv 0(\bmod 3)$. In these cases we have a biembedding of $C a y\left[\mathbb{Z}_{8 n+t} \oplus \mathbb{Z}_{d}: \pm \mathcal{E}(E)\right]$ in an orientable surface whose faces classes contain quadrangles and exactly one pentagon.

An example of such construction is given in Figure 6 where we provide a globally simple Archdeacon array over $\mathbb{Z}_{60} \oplus \mathbb{Z}_{d}$, where $d \geq 3$.

\section{ORCID iDs}

Simone Costa (D) https://orcid.org/0000-0003-3880-6299

Anita Pasotti (D) https://orcid.org/0000-0002-3569-2954

Marco Antonio Pellegrini (D) https://orcid.org/0000-0003-1742-1314 


\begin{tabular}{|c|c|c|c|c|c|c|}
\hline$(25,1)$ & $(0,-1)$ & & & $(1,0)$ & $(-8,0)$ & $(-18,0)$ \\
\hline$(-19,-1)$ & $(26,1)$ & & & & $(2,0)$ & $(-9,0)$ \\
\hline$(-10,0)$ & $(-20,0)$ & $(27,0)$ & & & & $(3,0)$ \\
\hline$(4,0)$ & $(-11,0)$ & $(-21,0)$ & $(28,0)$ & & & \\
\hline & $(5,0)$ & $(-12,0)$ & $(-22,0)$ & $(29,0)$ & & \\
\hline & & $(6,0)$ & $(-13,0)$ & $(-16,0)$ & $(23,0)$ & \\
\hline & & & $(7,0)$ & $(-14,0)$ & $(-17,0)$ & $(24,0)$ \\
\hline
\end{tabular}

Figure 6: An Archdeacon array over $\mathbb{Z}_{60} \oplus \mathbb{Z}_{d}$.

\section{References}

[1] B. Alspach and G. Liversidge, On strongly sequenceable abelian groups, Art Discrete Appl. Math. 3 (2020), \#P1.02 (19 pages), doi:10.26493/2590-9770.1291.c54.

[2] D. Archdeacon, Heffter arrays and biembedding graphs on surfaces, Electron. J. Combin. 22 (2015), \#P1.74 (14 pages), doi:10.37236/4874.

[3] D. S. Archdeacon, T. Boothby and J. H. Dinitz, Tight Heffter arrays exist for all possible values, J. Combin. Des. 25 (2017), 5-35, doi:10.1002/jcd.21520.

[4] D. S. Archdeacon, J. H. Dinitz, D. M. Donovan and E. Ş. Yazıcı, Square integer Heffter arrays with empty cells, Des. Codes Cryptogr. 77 (2015), 409-426, doi:10.1007/s10623-015-0076-4.

[5] D. S. Archdeacon, J. H. Dinitz, A. Mattern and D. R. Stinson, On partial sums in cyclic groups, J. Combin. Math. Combin. Comput. 98 (2016), 327-342.

[6] M. Buratti, Cycle decompositions with a sharply vertex transitive automorphism group, Matematiche (Catania) 59 (2004), 91-105, https: // lematematiche.dmi.unict. it/index.php/lematematiche/article/view/164.

[7] M. Buratti and A. Pasotti, Graph decompositions with the use of difference matrices, Bull. Inst. Combin. Appl. 47 (2006), 23-32.

[8] M. Buratti and A. Pasotti, On perfect $\Gamma$-decompositions of the complete graph, J. Combin. Des. 17 (2009), 197-209, doi:10.1002/jcd.20199.

[9] K. Burrage, D. M. Donovan, N. J. Cavenagh and E. Ş. Yazıcı, Globally simple Heffter arrays $H(n ; k)$ when $k \equiv 0,3(\bmod 4)$, Discrete Math. 343 (2020), 111787 (17 pages), doi:10. 1016/j.disc.2019.111787.

[10] N. J. Cavenagh, J. H. Dinitz, D. M. Donovan and E. Ş. Yazıc1, The existence of square noninteger Heffter arrays, Ars Math. Contemp. 17 (2019), 369-395, doi:10.26493/1855-3974. 1817.b97.

[11] N. J. Cavenagh, D. Donovan and E. Ş. Yazıcı, Biembeddings of cycle systems using integer Heffter arrays, J. Combin. Des (2020), doi:10.1002/jcd.21753.

[12] S. Costa, M. Dalai and A. Pasotti, A tour problem on a toroidal board, Australas. J. Combin. 76 (2020), 183-207, https: / / a jc.maths.uq.edu.au/pdf/76/ajc_v76_p183. pdf.

[13] S. Costa, F. Morini, A. Pasotti and M. A. Pellegrini, Globally simple Heffter arrays and orthogonal cyclic cycle decompositions, Australas. J. Combin. 72 (2018), 549-593, https: //ajc.maths.uq.edu.au/pdf/72/ajc_v72_p549.pdf.

[14] S. Costa, F. Morini, A. Pasotti and M. A. Pellegrini, A problem on partial sums in abelian groups, Discrete Math. 341 (2018), 705-712, doi:10.1016/j.disc.2017.11.013.

[15] S. Costa, F. Morini, A. Pasotti and M. A. Pellegrini, A generalization of Heffter arrays, $J$. Combin. Des. 28 (2020), 171-206, doi:10.1002/jcd.21684. 
[16] J. H. Dinitz and A. R. W. Mattern, Biembedding Steiner triple systems and $n$-cycle systems on orientable surfaces, Australas. J. Combin. 67 (2017), 327-344, https : / / a j c . maths . uq. edu.au/pdf/67/ajc_v67_p327.pdf.

[17] J. H. Dinitz and I. M. Wanless, The existence of square integer Heffter arrays, Ars Math. Contemp. 13 (2017), 81-93, doi:10.26493/1855-3974.1121.fbf.

[18] J. L. Gross and T. W. Tucker, Topological Graph Theory, Wiley-Interscience Series in Discrete Mathematics and Optimization, John Wiley \& Sons, New York, 1987.

[19] J. Hicks, M. A. Ollis and J. R. Schmitt, Distinct partial sums in cyclic groups: polynomial method and constructive approaches, J. Combin. Des. 27 (2019), 369-385, doi:10.1002/jcd. 21652.

[20] B. Mohar, Combinatorial local planarity and the width of graph embeddings, Canad. J. Math. 44 (1992), 1272-1288, doi:10.4153/cjm-1992-076-8.

[21] B. Mohar and C. Thomassen, Graphs on Surfaces, Johns Hopkins Studies in the Mathematical Sciences, Johns Hopkins University Press, Baltimore, Maryland, 2001.

[22] F. Morini and M. A. Pellegrini, On the existence of integer relative Heffter arrays, Discrete Math. 343 (2020), 112088 (22 pages), doi:10.1016/j.disc.2020.112088.

[23] M. A. Ollis, Sequences in dihedral groups with distinct partial products, Australas. J. Combin. 78 (2020), 35-60, https://ajc.maths.uq.edu.au/pdf/78/ajc_v78_ p035.pdf. 